\title{
Pharmacological Inhibition of STAT3 Attenuates Pancreatic Cancer Cell Invasion and Migration By Interacting with TGF-B/Smad Signaling
}

\section{hangcheng guo}

Wenzhou Medical University First Affiliated Hospital: The First Affiliated Hospital of Wenzhou Medical University

\section{yanyi xiao}

Wenzhou Medical University First Affiliated Hospital: The First Affiliated Hospital of Wenzhou Medical University

\section{Xuejia Yang}

Wenzhou Medical University First Affiliated Hospital: The First Affiliated Hospital of Wenzhou Medical University

\section{Ziwei Yuan}

Wenzhou Medical College First Affiliated Hospital: The First Affiliated Hospital of Wenzhou Medical University

\section{Qinbo Chen}

Wenzhou Medical University First Affiliated Hospital: The First Affiliated Hospital of Wenzhou Medical University

\section{Jiawei Chen}

Wenzhou Medical College First Affiliated Hospital: The First Affiliated Hospital of Wenzhou Medical University

\section{Chaoyue Chen}

Wenzhou Medical College First Affiliated Hospital: The First Affiliated Hospital of Wenzhou Medical University

\section{Mengsi Wang}

Wenzhou Medical College First Affiliated Hospital: The First Affiliated Hospital of Wenzhou Medical University

\section{Lumeng Shang}

Wenzhou Medical College First Affiliated Hospital: The First Affiliated Hospital of Wenzhou Medical University

\section{Lili Xie}

Wenzhou Medical University First Affiliated Hospital: The First Affiliated Hospital of Wenzhou Medical University

Qiyu Zhang 
Wenzhou Medical College First Affiliated Hospital: The First Affiliated Hospital of Wenzhou Medical University

\section{Yongheng Bai ( $\nabla$ wzbyh@wmu.edu.cn )}

Wenzhou Medical University First Affiliated Hospital: The First Affiliated Hospital of Wenzhou Medical University

\section{Research}

Keywords: Pancreatic ductal adenocarcinoma, epithelial-mesenchymal transition (EMT), STAT3, TGF$\beta /$ Smad

Posted Date: November 16th, 2021

DOI: https://doi.org/10.21203/rs.3.rs-1055773/v1

License: (c) (1) This work is licensed under a Creative Commons Attribution 4.0 International License. Read Full License 


\section{Abstract \\ Background}

Pancreatic ductal adenocarcinoma (PDAC) is a gastrointestinal malignancy with extremely poor prognosis, primarily due to the infiltration and metastasis of tumor cells. Many studies have shown that the TGF- $\beta$ /Smad signaling-mediated EMT pathway is a key driving factor of infiltration and metastasis of pancreatic cancer cells, and aberrant STAT3 activation is vital to pancreatic cancer cell metastasis. In this study, a small molecule antagonist, Stattic, was used for targeted STAT3 inhibition, its interaction with TGF- $\beta /$ Smad signaling was observed, and EMT of pancreatic cancer cells was inhibited to decrease its metastasis and infiltration.

\section{Methods}

The effects of Stattic on the invasion, infiltration, and EMT of pancreatic cancer cells were studied. We also evaluated the effects of Stattic on the STAT3 and TGF- $\beta$ /Smad signaling pathways and studied the interactions between STAT3 and TGF- $\beta /$ Smad signaling using overexpression and siRNA techniques.

\section{Results}

Stattic inhibited the invasion and infiltration of pancreatic cancer cells in a dose- and time-dependent manner, which was related to EMT inhibition and subsequent downregulation of extracellular matrix components. Stattic downregulated the expression of metalloproteases, such as MMP-2, and also inhibited the expression of EMT-TFs, such as Snail1 and Slug, and downregulated downstream HIF1a and VEGF expression to inhibit angiogenesis. Mechanistically, in in vitro cell models and in vivo nude mouse tumorigenicity models, Stattic treatment significantly downregulated the protein levels of the matrix synthesis-promoting factor TGF- $\beta$ and the phosphorylation and nuclear import of its downstream Smad2/3. These results suggest that Stattic inactivates TGF- $\beta /$ Smad signaling to antagonize EMT and eventually inhibit the invasion and infiltration of pancreatic cancer cells. Stattic is a small molecule inhibitor of STAT3 that can downregulate STAT3 activity, which is enhanced by Smad4-dependent TGF$\beta /$ Smad signaling. Interestingly, IL- 6 or STAT3 overexpression promotes EMT but in a non-TGF- $\beta /$ Smaddependent manner, and is regulated by antagonism between STAT3 and Smad4.

\section{Conclusions}

The application of Stattic pharmacologically to inhibit STAT3 decreases the invasion and infiltration of pancreatic cancer cells, which may be achieved through interactions with TGF- $\beta /$ Smad signaling and EMT antagonism. 


\section{Background}

Pancreatic cancer is a highly malignant gastrointestinal tumor with an extremely poor prognosis. Epidemiological data show that the 5-year survival rate of pancreatic cancer patients is less than $8 \%$ [1]. At present, surgery is considered as the mainstay method for treating pancreatic cancer. However, most patients will miss the opportunity for surgery due to invasion and metastasis of pancreatic cancer cells. Even after surgery, extensive dissemination of metastases results in poor efficacy and prognosis [2-4]. Therefore, examining the invasion and metastasis capacity of pancreatic cancer cells may be key in determining patient prognosis.

Pancreatic ductal adenocarcinoma (PDAC), a ductal differentiated invasive tumor of epithelial origin, is the most common type of pancreatic cancer, accounting for $80-90 \%$ of pancreatic cancers, and is characterized by significant interstitial fibrous tissue hyperplasia [5, 6]. Epithelial-mesenchymal transition (EMT) is one of the main causes driving the invasion and metastasis of PDAC [7]. During EMT, pancreatic ductal cells with epithelial characteristics undergo induction by KRAS or mutant TP53 $[8,9]$, or stimulation by external injury factors, such as hypoxia and various growth factors. This results in the loss of epithelial cell markers, such as E-cadherin and ZO-1, and leads the cells to differentiate into mesenchymal cells, which highly express $\mathrm{N}$-cadherin and a-SMA and are more suited to growth in an injury microenvironment and have a tendency to migrate. Currently, it is believed that the activation of TGF- $\beta$ /Smad is one of the factors driving EMT induction $[10,11]$. TGF- $\beta$ binds to its receptor TGF- $\beta$ R 1 and affects TGF- $\beta$ R2 phosphorylation. The latter promotes phosphorylation of the carboxy terminal of the intracellular signal effector proteins Smad2 and Smad3 (canonical pathway) to form a Smad multimeric complex under the action of cytoplasmic Smad. This complex enters the nucleus and binds to specific regulatory elements on target genes to induce the transcription of downstream target genes and promote a series of biological behaviors, including EMT $[12,13]$. Whole genome sequencing has shown that loss of Smad 4 is present in $60 \%$ of pancreatic cancers. Loss of Smad 4 can cause a defect in TGF- $\beta / S$ Smad signaling and the activation of non-canonical TGF- $\beta$ signaling. Non-canonical TGF- $\beta$ signaling causes EMT through the RhoA, Ras, and MAP3K7 pathways [14-16]. Therefore, the presence or absence of Smad4 induces tumor cell EMT through different ways and leads to invasion and metastasis of pancreatic cancer cells.

Previous studies have shown that STAT3 activation is involved in EMT of pancreatic cancer cells. After IL6 stimulation, STAT3 is activated and can activate EMT-related transcription factors, such as Snail 1 and Snail2 (slug), to abrogate E-cadherin expression and promote the expression of $\mathrm{N}$-cadherin and a-SMA $[17,18]$. STAT3 activation also promotes EMT through coupling with TGF- $\beta /$ Smad signaling. Studies have shown that STAT3 selectively interacts with Smad3 to antagonize its binding with Smad4, thereby inhibiting nuclear import of its complex and ultimately antagonizing TGF- $\beta$ signal transduction $[19,20]$. STAT3 phosphorylation is also inhibited by Smad4, thereby blocking TGF- $\beta$-induced tumor cell infiltration and metastasis [21]. Regulating STAT3 activity, such as by using small molecule inhibitors, may be a potential strategy to inhibit pancreatic cancer metastasis. 
In this study, we evaluated the effects of the STAT3 small molecule antagonist, Stattic, on pancreatic cancer invasion and metastasis in in vivo and in vitro experiments. We also examined the crosstalk between STAT3 signaling and TGF- $\beta /$ Smad signaling in EMT. Our results showed that Stattic can block EMT, both by reducing TGF- $\beta$ /Smad signaling activity and Smad4-dependent STAT3 inactivation, to inhibit the invasion and infiltration of pancreatic cancer cells. Moreover, we found that a small molecule agonist or STAT3 overexpression reversed the antineoplastic effects of Stattic in a non-TGF- $\beta / S m a d-$ dependent manner. Therefore, Stattic antagonism of pancreatic cancer metastasis may be achieved by regulating crosstalk between STAT3 and TGF- $\beta /$ Smad pathways.

\section{Methods}

\section{Cell culture}

Pancreatic cancer cells (PANC-1, BxPc-3, Patu-8988, and CFPAC-1) and a normal pancreatic ductal epithelial cell line (hTERT-HPNE) were obtained from National Collection of Authenticated Cell Cultures (Shanghai, China). PANC-1, Patu-8988, and hTERT-HPNE cells were cultured in Dulbecco's Modified Eagle's Medium (DMEM, Gibco Inc, Invitrogen, USA). CFPAC-1 and BxPc-3 cells were grown in Roswell Park Memorial Institute-1640 medium (RPMI-1640, Invitrogen). Both types of media 10\% fetal bovine serum (FBS, Invitrogen), $100 \mathrm{U} \cdot \mathrm{ml}^{-1}$ penicillin, and $100 \mu \mathrm{g} \cdot \mathrm{ml}^{-1}$ streptomycin (Invitrogen) added as nutrient supplements and antibacterial drugs. The cells were cultured in a $37^{\circ} \mathrm{C}$ and $5 \% \mathrm{CO}_{2}$ cell culture incubator. Stattic used in the experiments was purchased from MCE (Fig. 1A, CAS No: 19983-44-9, MCE, China), as was human IL-6. Recombinant human TGF- $\beta 1$ was purchased from Peprotech (Suzhou, China).

\section{Lentivirus transfected cells and overexpression of Smad4}

The lentiviruses, plasmids, and negative control were designed and manufactured by Jikai Gene (Shanghai, China). The lentivirus operation manual of Jikai Gene was used as a reference for the experimental protocol. Briefly, PANC-1 cells were infected with lentiviruses (LV-STAT3,24120-1, Ubi-MCS3FLAG-CBh-gcGFP-IRES-puromycin) at a multiplicity of infection (MOI) of 10 and the negative control group was infected with the negative control lentivirus (CON355). The cells were seeded in a 6-well plate at a concentration of $1.5 \times 10^{5}$ of cells per well. When the cells showed adherent growth, the corresponding amount of lentiviruses and $40 \mu \mathrm{l}$ of virus infection reagent HitransG P (Jikai Gene) were added to increase the infection rate. After $24 \mathrm{~h}$ of lentivirus infection, the culture medium was changed to complete culture medium and the cells were cultured for a further 2 days. Subsequently, $1.5 \mu \mathrm{g} / \mathrm{ml}$ and $0.5 \mu \mathrm{g} / \mathrm{ml}$ puromycin were used to screen cells that were successfully infected with lentiviruses for subsequent experiments. For Smad4 overexpression in BxPc-3 cells, the overexpression plasmid sequence (SMAD4-F-Flag-Hind囚, 5'-

CGAAGCTTATGGACTACAAGGACGACGATGACAAGATGGACAATATGTCTATTACG A-3', and SMAD4-R-TGABamH囚, 5'-ATGGATCCTCAGTCTAAAGGTTGTGGGTCTGCA-3') was obtained from Sigma-Aldrich (Shanghai, China) and synthesized by Sangon (Shanghai, China). The plasmid was amplified and purified for subsequent experiments. Similarly, $1.5 \times 10^{5}$ of BxPc-3 seeds were seeded per well in a 6-well 
plate. After cells had adhered to the plate, $0.5 \mathrm{mg} / \mathrm{mL}$ plasmid was added and culture was continued for a further $24 \mathrm{~h}$. Next, $400 \mu \mathrm{g} / \mathrm{mL}$ G418 was used to screen Smad4 overexpressing cells for subsequent experiments.

\section{Cell migration}

The cell migration capacity was measured using scratch assays. Briefly, PANC-1 and BxPc-3 cells were cultured in 6-well plates for $48 \mathrm{~h}$. When the cells reached confluency, a pipette tip was used to make a scratch in the cell monolayer. The detached cells were washed with phosphate buffered saline (PBS) and an inverted microscope was used to take photographs on the first day of the scratch assay. Subsequently, complete culture medium and corresponding drugs were added and photographs were taken every $24 \mathrm{~h}$. The experiment was repeated in triplicate and ImageJ software was used to calculate the wound healing area.

\section{Cell invasion ability}

The Transwell invasion assay was used to measure the cell invasion ability. In brief, $200 \mu$ serum-free culture medium was used to resuspend $2 \times 10^{5}$ PANC- 1 or BxPc- 3 cells for addition to pre-coated Transwell chambers (Costar, NY, USA, \#3422) together with the corresponding drugs. Next, $600 \mu \mathrm{l}$ of complete culture medium containing $10 \%$ FBS was added to the lower chambers of a 24-well plate. After $24 \mathrm{~h}$, a sterile cotton swab was used to carefully scrape off cells on the upper surface of the Transwell chambers. The cells that migrated to the lower surface of the membrane were fixed with $4 \%$ paraformaldehyde (Solarbio, China) and stained with crystal violet (Solarbio). An inverted microscope was used to capture three fields and ImageJ software was used to enumerate the infiltrating cells.

\section{Immunofluorescence staining}

Immunofluorescence staining was conducted based on a previous protocol [22]. In brief, PANC-1 and BxPc-3 cells were adhered to glass slides and subjected to drug treatment for the corresponding durations. Next, $4 \%$ paraformaldehyde (Solarbio) was used to fix the cells. After fixing, $0.1 \%$ Triton X-100 was used to treat the cells and goat serum (BOSTER, Wuhan, China) was used to block non-specific binding. After blocking, cells were incubated with the corresponding primary antibodies at $4^{\circ} \mathrm{C}$ overnight. The antibodies used were as follows: N-cadherin (1:200, Abcam, Shanghai, China), E-cadherin (1:200, ProteinTech, Wuhan, China), a-SMA (1:200, Affinity Biosciences, Jiangsu, China), and Collagen $\otimes(1: 200$, Abcam), and Snail1 (1:200, Abcam), and Slug (1:200, Abcam), and TGF- $\beta 1$ (1:200, Affinity), and p-Smad2 (1:200, Affinity), and p-Smad3 (1:200, Affinity), and p-STAT3 (1:200, Abcam), and Smad4 (1:200, ProteinTech). Next, DyLight 488 (green fluorescence) or DyLight 594 (red fluorescence) conjugated goat anti-rabbit IgG antibodies (1:400, ProteinTech) were allowed to bind to primary antibodies. Finally, DAPI (Solarbio) was used to stain the cell nuclei and a fluorescence microscope was used to obtain fluorescence images.

\section{Animal experiments}


All animal experiments were approved by the Animal Research Ethics Committee of Wenzhou Medical University. Nude mice were housed in specific pathogen-free facilities with independent ventilation systems. Six mice were housed in one cage under a $12 \mathrm{~h}-12 \mathrm{~h}$ light-dark cycle at $24^{\circ} \mathrm{C}$. Standard food and drinking water was provided to the mice. During the experiment, $5 \times 10^{6}$ of PANC-1 cells were resuspended in $100 \mu \mathrm{l}$ PBS and injected subcutaneously into the backs of twelve 4-week old male nude mice (BALB/c, Wenzhou). The mice were then randomized into two groups. In the experimental group ( $\mathrm{n}=$ 6), Stattic $(10 \mathrm{mg} / \mathrm{kg})$ was administered every day by intraperitoneal injection for 14 days. In the control group $(n=6)$, the same volume of physiological saline was injected into the mice. The tumor diameter was measured daily and the tumor volume was calculated based on the following formula: $V=$ length $^{2} \times$ width. The weights of the mice were monitored. At the end of the experiment, the mice were euthanized using carbon dioxide asphyxiation. Mice were necropsied and their weights were recorded. The mice were photographed and the tumors were harvested. Tumor tissues used for sectioning were stored in $4 \%$ paraformaldehyde and tissues used for protein and RNA extraction were stored in liquid nitrogen.

\section{Histological analysis}

Paraformaldehyde-fixed tumor tissues were flushed with water, followed by dehydration, paraffin embedding, and sectioning. Immunohistochemistry (IHC, Solarbio) staining and hematoxylin and eosin (HE, Solarbio) staining were conducted after sections were processed. IHC staining was performed on 4 $\mu \mathrm{M}$-thick paraffin tissue sections. After clearing in xylene, the sections were soaked in different concentrations of ethanol. Next, the sections were heated in $0.1 \%$ sodium citrate buffer for antigen retrieval, before adding endogenous peroxide blocker (BOSTER) [23]. The following antibodies were used for IHC staining: N-cadherin (1:200, Abcam, Shanghai, China), a-SMA (1:200, Affinity), and collagen III (1:200, Abcam). The DAB color development kit (Zhongshan Golden Bridge Biotechnology, Beijing, China) was used for color development. HE staining was performed according to the instruction manual of the reagent. A DM4000B LED Microscope System (Leica Microsystems) and a DFC 420C 5M Digital Microscope Camera (Leica Microsystems) were used to observe and photograph the IHC- and HE-stained sections.

\section{Quantitative real-time PCR (qRT-PCR)}

A SCIENTZ-48 high-throughput tissue homogenizer (Xinzhi Bio-technology and science Inc, Ningbo, China) was used to homogenize tumor tissues at low temperature, and RNAiso Plus (TaKaRa, Dalian, China) was used to extract total RNA. PANC-1 and BxPc-3 cells were cultured in 6-well plates and RNAiso Plus was used to extract total RNA. The purity and concentration of the total extracted RNA was measured before using the PrimeScript RT reagent kit (Perfect Real Time) (TaKaRa) for reverse transcription of total RNA to CDNA. Next, DNase and RNase free DEPC water (Biosharp, Hefei, China) were added to prepare identical cDNA concentrations. SYBR Green (Toyobo, Osaka, Japan) and corresponding primers were used for qRT-PCR of cDNA in an ABI 7500Fast Real-Time PCR system (Applied Biosystems, Foster City, CA, USA). Simultaneously, the melt curve of the primers was monitored to validate primer specificity. B-actin was used as theinternal reference gene and the $\triangle \triangle C T$ method was used to obtain the 
relative mRNA expression level [24]. The experiment was repeated in triplicate and the primers were synthesized by Sangon (Sangon). The forward and reverse primer sequences: $\beta$-actin, $5^{\prime}-$

TGACGTGGACATCCGCAAAG-3' and 5'-CTGGAAGGTGGACAGCGAGG-3', Col1a1, 5'-

TGGCAAAGAAGGCGGCAAAGG-3' and 5'-AGGAGCACCAGCAGGACCATC-3', Cdh2, 5'-

AGGAGTCAGTGAAGGAGTCAGCAG-3' and 5'-TTCTGGCAAGTTGATTGGAGGGATG-3', STAT3, 5'-

GAGGCAGGAGAATCGCTTGAACC-3' and 5'-TCTCAGACTGTCGCCCAGGAT G-3'.

\section{Immunoblot analyses}

A tissue homogenizer was used to homogenize pancreatic cancer tissues. Simultaneously, EDTA-free protease, phosphatase inhibitor tablets, and T-PER tissue protein extraction reagent (all Thermo Fisher Scientific) were added to extract total protein. The same reagents were used for lysis and total protein extraction from PANC-1 and BxPc-3 cells. A BCA protein concentration assay kit (Beyotime, Shanghai, China) was used to measure the concentration of total protein. Next, $20 \mu \mathrm{g}$ of processed total protein was loaded onto a 10\% SDS-PAGE gel for electrophoresis. Polyvinylidene fluoride (PVDF) membranes (Sigma Aldrich) were used as the transfer membranes. The membranes were blocked with $5 \%$ skimmed milk before incubation with primary antibodies: N-cadherin (1:1000, Abcam), E-cadherin (1:1000, ProteinTech), a-SMA (1:1000, Affinity), and Vimentin (1:1000, Affinity), and MMP2 (1:1000, ProteinTech), and MMP7 (1:1000, ProteinTech), and MMP9 (1:1000, ProteinTech), and HIF-1a (1:1000, Abcam), and VEGF (1:1000, Abcam), JAK2 (1:1000, Abcam), and p-JAK2 (1:1000, Abcam), and STAT3 (1:1000, Abcam), and p-STAT3 (1:1000, Abcam), and Collagen $\otimes(1: 1000$, Abcam), and Collagen $\otimes(1: 1000$, Abcam), and Snail1 (1:1000, Abcam), and Slug (1:1000, Abcam), and TGF- $\beta 1$ (1:1000, Affinity), and Smad2/3 (1:1000, Affinity), and p-Smad2 (1:1000, Affinity), and p-Smad3 (1:1000, Affinity), and Smad4 (1:1000, ProteinTech), and GAPDH (1:2000, Abcam) at $4^{\circ} \mathrm{C}$ overnight. GAPDH was used as the internal reference. On the following day, horseradish peroxidase (HRP)-conjugated secondary antibodies were allowed to bind to primary antibodies. The SuperSignal West Pico Chemiluminescent Substrate (Thermo Fisher Scientific) device was used to visualize the results [25]. Finally, ImageJ software was used to calculate the grayscale values of western blots.

\section{Statistical analysis}

The experimental results are expressed as the mean and standard deviation. GraphPad Prism software 8.0 (GraphPad Software, Inc., La Jolla, CA, USA) was used to perform the statistical analysis of data. Two-sided Student's t-test was used for statistical analysis when there were two experimental groups, and one-way analysis of variance (ANOVA) was used for statistical comparison when there were more than two experimental groups. A difference of $\mathrm{P}<0.05$ was considered statistically significant.

\section{Results}

\section{Stattic inhibits the invasion and migration of PCCs}


From scratch assays, we observed that $4 \mu \mathrm{M}$ Stattic inhibited PANC-1 migration following 24-h culture (inhibition rate of $50.5 \%$ ), while a higher concentration $(10 \mu \mathrm{M})$ of Stattic showed greater inhibitory effects ( $96.2 \%$, Fig. 1B). Even though PANC-1 migration was evident after $48 \mathrm{~h}$ of culture, Stattic still exhibited some inhibitory effects ( $40.9 \%$ for 24 h, $92.1 \%$ for 48 h, Fig. 1B and C). Stattic also exhibited inhibitory effects in another tumor cell line, BxPc-3. Even though these effects were weaker than those observed in PANC-1 cells, more than $50 \%$ inhibition was achieved at a concentration of $10 \mu \mathrm{M}$ (Fig. 1D and E). We also studied the effects of Stattic on pancreatic cancer cell invasion. As shown in Fig. 1F-H, Stattic significantly decreased the number of invading PANC-1 $(72.5 \%$ for $4 \mu \mathrm{M}, 87.8 \%$ for $10 \mu \mathrm{M}$,) and BxPc-3 $(59.4 \%$ for $4 \mu \mathrm{M}, 70.9 \%$ for $10 \mu \mathrm{M})$ cells. These findings demonstrate that Stattic inhibits the invasion and migration of pancreatic cancer cells in a concentration-dependent manner.

\section{Stattic inhibits the expression of EMT-related proteins and MMPs, and promotes extracellular matrix (ECM) accumulation in PCCs}

Many studies have pointed out that EMT is a key driving factor of invasion and migration of tumor cells $[26,27]$. Therefore, we also analyzed effects of Stattic on EMT in different pancreatic cancer cell lines. Stattic did not significantly inhibit the expression of the cytoskeleton protein vimentin in PANC-1 and BxPc-3 cells (Fig. 2A and B). However, Stattic significantly upregulated the expression of the epithelial cell marker E-cadherin, and downregulated the expression of the mesenchymal cell markers $\mathrm{N}$-cadherin and aSMA (Fig. 2A-C). This shows that Stattic has some inhibitory effects toward EMT. Stattic treatment also resulted in a decrease in the secretion of ECM components, such as type I and type III collagen, in the mesenchymal tumor cells (Fig. 2D-F). We also observed that the expression of metalloproteases, which that regulate the invasion and migration of tumor cells (including MMP-2 and MMP-9) were inhibited by Stattic (Fig. $2 \mathrm{G}$ and $\mathrm{H}$ ), further validating the inhibitory effects of Stattic on the invasion and migration of tumor cells $[28,29]$.

\section{Stattic inhibits the expression of EMT-TFs and VEGF in PCCs}

The phenotypic transformation of tumor cells is regulated by transcription factors such as Snail1 and Snail2 (Slug) [30]. Therefore, we also investigated the effects of Stattic on Snail1 and Slug expression. As shown in Fig. 3A and B, Stattic significantly inhibited both Snail1 and Slug expression.

Immunofluorescence staining also showed that cytoplasmic Snail1 and Slug expression were inhibited in PANC-1 and BxPc-3 cells (Fig. 3C-F). This finding suggests that inhibition of EMT in tumor cells may be due to inhibition of Snail1 and Slug expression. Tumor neovascularization is another important factor that affects tumor cell metastasis [31, 32]. Therefore, we also assessed the effects of Stattic on angiogenesis proteins. We found that Stattic not only inhibits VEGF expression but also inhibits the expression of HIF-1a, a protein related to the hypoxic tumor microenvironment (Fig. 3G and H). These findings show that Stattic inhibits EMT by antagonizing EMT-TFs. Simultaneously, Stattic inhibits the HIF$1 \mathrm{a}-\mathrm{VEGF}$ axis to antagonize tumor angiogenesis [33].

\section{Stattic antagonizes TGF- $\beta 1$ expression and downstream Smad signal activation}


Previous studies have reported that TGF- $\beta 1$ and the downstream Smad signaling cascade participates in the transcriptional regulation of EMT-TFs [34,35]. In this study, we observed significant differences in TGF- $\beta 1$ expression levels between different tumor cells (Additional file 1: Fig. S1), and the TGF- $\beta 1$ expression levels in PANC-1 and BxPc-3 cells were significantly higher than that in normal pancreatic ductal epithelial cells (hTERT-HPNE). Stattic downregulated TGF- $\beta 1$ expression in PANC-1 and BxPc-3 cells (Fig. 4A and B). In tumor cells, TGF- $\beta 1$ phosphorylates cytoplasmic Smad2 and Smad4 through its membrane receptor so that these two proteins enter the nucleus and binds to target genes to induce the expression of downstream EMT-TF Snail1. We observed that Stattic treatment significantly decreased the phosphorylation levels of Smad2 (T220) and Smad3 (S423/425) in PANC-1 and BxPc-3 cells and inhibited their nuclear import (Fig. 4C-E). This evidence shows that targeted inhibition of the TGF$\beta 1 /$ Smad signaling cascade may be one of the important factors by which Stattic antagonizes EMT.

\section{Stattic inhibits the growth of PDAC and antagonizes TGF- $\beta 1 /$ Smad signaling in an in vivo nude mouse tumorigenesis model}

In vitro studies have demonstrated that Stattic has inhibitory effects on the invasion and infiltration of pancreatic cancer cells. We next constructed a nude mouse model to evaluate the in vivo antineoplastic effects of Stattic (Fig. 5A). In this model, the weight (Fig. 5B) and volume (Fig. 5C) of pancreatic tumors significantly decreased after Stattic treatment. HE staining demonstrated that Stattic resulted in fibrosis inhibition in tumor cells (Fig. 5D). Moreover, the qRT-PCR results showed that the fibrosis-related gene Col1a1 (encodes type I collagen) and the EMT-related Cdh2 (encodes N-cadherin) were significantly downregulated after Stattic treatment (Fig. 5E) $[35,36]$. Similarly, the results of IHC and western blot demonstrated that Stattic downregulated the expression of a-SMA, N-cadherin, and type I collagen to inhibit EMT in the nude mouse tumorigenicity model (Fig. 5F-H). These findings show that Stattic inhibited the activation of the TGF- $\beta 1 /$ Smad signaling cascade in pancreatic cancer cells. The nude mouse tumorigenicity model demonstrated that TGF- $\beta 1$ expression was inhibited by Stattic (Fig. $5 \mathrm{I}$ and J), which downregulated Smad2/3 phosphorylation (Fig. 5I and J). Therefore, these findings show that Stattic antagonizes TGF- $31 /$ Smad signaling to inhibit tumor growth and EMT in the nude mouse tumorigenicity model.

\section{Stattic-mediated inhibition of PCC invasion and migration is reversed by TGF- $\beta 1$ treatment}

The above in vivo and in vitro experiments revealed that EMT antagonism by Stattic is achieved by antagonizing the activation of the TGF- $\beta 1 /$ Smad signaling cascade. We next sought to investigate whether the activation of TGF- $\beta 1 /$ Smad signaling reverses the anti-pancreatic cancer metastasis effects of Stattic. First, at the morphology level, TGF- $\beta 1$ treatment for $24 \mathrm{~h}$ was found to reverse the decrease in fibrosis-like tumor cells caused by Stattic (Fig. 6A). Secondly, scratch assays showed that TGF- $\beta 1$ antagonized Stattic-induced inhibition of migration of PANC-1 and BxPc-3 cells (Fig. 6B), while TGF- $\beta 1$ blocked the Stattic-induced reduction of tumor cell invasion (Fig. 6C). Mechanistically, TGF- $\beta 1$ activates Smad2/3 signaling (Fig. 6D and E) to inhibit Stattic-mediated downregulation of EMT-TFs (Snail1 and Slug) (Fig. 6F), thereby causing TGF- $\beta 1$ to promote EMT in PANC-1 and BxPc-3 cells (Fig. 6G and H). 
Therefore, TGF- $\beta 1$ reverses Stattic-induced inhibition of pancreatic cancer cell metastasis through downstream Smad2/3 signaling.

\section{Stattic-induced STAT3 inactivation in PCCs is exacerbated by Smad4-dependent TGF- $\beta 1$ signaling}

The above evidence shows that Stattic has the potential to be used as a small molecule inhibitor of STAT3 [37]. Therefore, we also assessed the effects of Stattic on STAT3 activity in PANC-1 and BxPc-3 cells. In PANC-1 cells, we found that Stattic simultaneously inhibited the phosphorylation of JAK2 (Tyr1007/1008) and STAT3 (Y705) (Fig. 7A and B) and antagonized the nuclear import of STAT3 (Fig. 7C). Interestingly, Stattic did not significantly downregulate JAK2 and STAT3 phosphorylation in BxPc-3 cells (Fig. 7A-C), which may be related to a significantly higher STAT3 activity in BxPc-3 cells than in PANC-1 cells (Fig. 7D). Furthermore, we observed an interesting phenomenon in that Stattic downregulation of STAT3 activity in PANC-1 and BxPc-3 cells was further exacerbated by TGF- $\beta 1$ treatment (Fig. 7E and F). Previous papers have reported that Smad4 could antagonize STAT3 phosphorylation, thereby antagonizing TGF- $\beta 1$-induced invasion and infiltration of pancreatic cancer cells [21]. Therefore, we deduced that TGF- $\beta 1$-induced STAT3 inactivation may be related to Smad4 activation. First, we assessed whether Stattic could inhibit Smad4 activity in pancreatic cancer cells. The expression of Smad4 varies across pancreatic cancer cell lines, Smad4 expression was increased in PANC-1 cells but was not expressed in BxPc-3 cells (Additional file 2: Fig. S2) [38]. After Stattic treatment, Smad4 expression was significantly downregulated in PANC-1 cells (Additional file 3: Fig. S3A) and nuclear translocation was inhibited in parallel (Additional file 3: Fig. S3B). Secondly, we found that Statticmediated Smad4 activity inhibition was abolished in PANC-1 cells after TGF- $\beta 1$ treatment (Additional file 3: Fig. S3C and D). Thirdly, Smad4 overexpression in BxPc-3 cells inhibited STAT3 phosphorylation and nuclear localization (Additional file 4: Fig. S4A and B). These findings show that Stattic induced STAT3 inactivation in pancreatic cancer cells, and that STAT3 inactivation is induced by TGF- $\beta 1$ through activating Smad4-dependent mechanisms.

\section{Activating STAT3 abolishes Stattic-mediated inhibition of PCC invasion and migration independent of TGF- $\beta /$ Smad signaling}

Assuming that STAT3 inactivation participates in Stattic-induced inhibition of pancreatic cancer cell metastasis, we next sought to determine whether activating STAT3 reverses the antineoplastic effects of Stattic. First, we treated cells using IL-6, a STAT3 agonist [39]. As expected, IL-6 not only induced JAK2 phosphorylation in PANC-1 and BxPc-3 cells after Stattic treatment, but also promoted STAT3 phosphorylation (Fig. 8A). This suggests that the JAK2/STAT3 signaling that was downregulated by Stattic was reactivated in pancreatic cancer cells. IL-6-induced STAT3 activation causes an increase in tumor cell migration and infiltration (Fig. 8B-E). This increase in migration capacity is related to IL-6 reversal of Stattic-mediated EMT inhibition (Fig. 8F). Interestingly, IL-6 did not increase the TGF- $\beta 1$ level (Fig. 8G), nor did it induce downstream Smad2/3 phosphorylation and nuclear import (Fig. 8G and H), suggesting that inactivation of TGF- $\beta 1 /$ Smad signaling caused by Stattic could not be reversed by IL-6. Therefore, IL-6 antagonizes Stattic-induced JAK2/STAT3 signaling inactivation to abolish the inhibitory 
effects of Stattic on EMT and tumor cell migration independent of Stattic-induced TGF- $\beta 1 /$ Smad signaling.

We next conducted overexpression studies in PANC-1 cells with weak STAT3 activity. We found that overexpressing STAT3 without changing its phosphorylation or nuclear transcription (Fig. 9A and Additional file 5: Fig. S5) still antagonized Stattic-induced inhibition of PANC-1 migration and infiltration (Fig. 9B and C). These mechanisms are similar to those of IL-6, i.e., Stattic-induced EMT inhibition is abolished by STAT3 overexpression (Fig. 9D-F). Moreover, STAT3 overexpression did not increase TGF$\beta 1 /$ Smad signaling activity (Fig. $9 \mathrm{G}$ and H). Previous studies have shown that STAT3 can selectively bind to Smad3 to decrease binding between Smad3 and Smad4, thereby inhibiting nuclear import of its complex and ultimately antagonizing TGF- $\beta 1$ signal transduction [40]. Our study also shows that IL-6 stimulation or STAT3 overexpression in PANC-1 cells did not abolish Stattic-mediated inhibition of Smad4 nuclear import (Additional file 6: Fig. S6A-D). In summary, these findings show that although the STAT3 small molecule inhibitor Stattic could simultaneously antagonize STAT3 and TGF- $\beta /$ Smad signaling activity to inhibit EMT, infiltration, and invasion in pancreatic cancer cells, the EMT promoted by STAT3 small molecule agonist or STAT3 overexpression occurs through a non-TGF- $\beta$ /Smad-dependent mechanism. These results demonstrate that there are crosstalk mechanisms between STAT3 and TGF$\beta /$ Smad signaling during pancreatic cancer metastasis.

\section{Discussion}

In this paper, Stattic was shown to inhibit the invasion and infiltration of pancreatic cancer cells in a doseand time-dependent manner. Further study found that Stattic downregulated the expression of metalloproteases, such as MMP-2, and inhibited the activity of EMT-TFs, such as Snail1 and Slug, to antagonize EMT and decrease ECM components [41]. Stattic also decreased HIF-1a and VEGF expression, showing that it may have some inhibitory effects on tumor angiogenesis [42]. In addition, Stattic could inhibit pancreatic cancer growth and EMT in an in vivo nude mouse tumorigenicity model. These findings showed that Stattic has potential to be used as a drug for treating pancreatic cancer.

Stattic is an effective STAT3 inhibitor that inhibits STAT3 phosphorylation (phosphorylation sites are Y705 and S727). Stattic inhibits binding between high affinity phosphopeptides and the SH2 domain of STAT3 [37]. Stattic has potential antineoplastic effects and inhibits glutathione reductase through a ROSdependent pathway to inhibit tumorigenicity in human cervical cancer cells $[43,44]$. Stattic has also been shown to inhibit STAT3 to antagonize bone metastasis in prostate cancer [45]. Moreover, Stattic has previously been shown to possess anti-pancreatic cancer activity, which is consistent with the results of our study [46]. Mechanistically, our study also showed that Stattic not only downregulates STAT3 phosphorylation and nuclear import in PANC-1 cells to inhibit its activity, but can also inhibit the phosphorylation and nuclear import of upstream JAK2. Interestingly, Stattic does not inhibit JAK2/STAT3 signaling activity in BxPc-3 cells. We observed that STAT3 activity in BxPc-3 cells is significantly higher than PANC-1 cells; therefore, we speculated that insignificant inhibition of STAT3 activity may be due to an insufficient dose of Stattic. However, high concentrations of Stattic induced significant rates of cell 
death, which is detrimental to treatment. Therefore, the antineoplastic effects of a suitable concentration of Stattic in tumor cells with high STAT3 activity may not only depend on STAT3 mechanisms.

TGF- $\beta /$ Smad signaling is the most important factor involves in EMT $[47,48]$. In pancreatic cancer, aberrant TGF- $\beta$ /Smad signaling activation induces the expression of EMT-TFs to drive EMT, thereby promoting tumor cell invasion and infiltration [49]. Our in vitro experimental results showed that Stattic significantly inhibited TGF- $\beta /$ Smad signaling in two pancreatic cancer cell lines. Similarly, the in vivo nude mouse tumorigenicity model also showed that TGF- $\beta /$ Smad signaling significantly decreased after Stattic treatment. Decreased TGF- $\beta /$ Smad signaling activity not only presents as reduced Smad 2 and Smad3 phosphorylation and blockade of nuclear import, but also inhibits Smad4 expression and nuclear import in Smad4-positive PANC-1 cells. TGF- $\beta$ stimulation could reverse Stattic-induced inhibition of pancreatic cancer cell metastasis through downstream Smad2/3 signaling. Additionally, TGF- $\beta$ could restore Smad4 activity that was downregulated by Stattic in PANC-1 cells. These findings show that the antineoplastic effects of Stattic are independent of TGF- $\beta /$ Smad signaling inactivation.

As mentioned above, Stattic inhibits JAK2/STAT3 signaling and TGF- $\beta$ /Smad signaling. We next sought to investigate whether there was a relationship between the two signaling pathways $[50,51]$. We observed that Stattic downregulation of STAT3 activity could be enhanced by TGF- $\beta$. Interestingly, TGF- $\beta$-induced Smad4 hyperactivity was significantly related to STAT3 downregulation. Smad4 expression in Smad4negative BxPc-3 cells was found to significantly inhibit STAT3 activity, demonstrating antagonism between Smad4 and STAT3. Previous studies have shown that STAT3 could block binding between Smad4 and Smad3 to inhibit its nuclear translocation [52]. In contrast, Smad4 has been shown to inhibit STAT3 phosphorylation to block its signal transduction [21]. Our results demonstrated that treatment with the JAK2/STAT3 agonist, IL-6, or STAT3 overexpression could abolish Stattic-induced EMT inhibition, although this occurs through a non-TGF- $\beta /$ Smad-dependent mechanism. Further results showed that the Stattic-induced reduction in Smad4 expression and nuclear import in PANC-1 cells was not reversed by IL6 treatment or STAT3 overexpression; this demonstrated that STAT3 activation may be detrimental to Smad4 nuclear import and is unable to promote intracellular TGF- $\beta /$ Smad signal transduction.

Our study also has some limitations. First, the mechanism by which Stattic directly inhibits TGF- $\beta /$ Smad signaling requires further validation. Secondly, an in vivo gene knockout model may be required to determine the crosstalk between STAT3 and TGF- $\beta /$ Smad signaling. More importantly, as Stattic is a potential antineoplastic drug in clinical practice, more in-depth studies are necessary to assess the optimal dose, safety, and efficacy of Stattic.

\section{Conclusion}

In summary, our in vivo and in vitro studies demonstrated the I anti-invasion and infiltration effects of the STAT3 small molecule inhibitor Stattic on pancreatic cancer cells. Stattic inhibits STAT3 signaling and activation of TGF- $\beta /$ Smad signaling to block EMT of pancreatic cancer cells. Mechanistically, STAT3 and 
Smad4 are antagonistic to each other, thereby regulating JAK2/STAT3 signaling or TGF- $\beta /$ Smad signaling activity and eventually affecting pancreatic cancer invasion and infiltration through EMT.

\section{Abbreviations}

DAPI, 4'6-diamidino-2-phenylindole, DMEM, Dulbecco modified Eagle medium, RPMI-1640, Roswell Park Memorial Institute-1640 medium, EMT, epithelial mesenchymal transition, FBS, fetal bovine serum, PBS, phosphate buffered saline, IHC, Immunohistochemistry, HE, hematoxylin and eosin, PVDF, Polyvinylidene fluoride, GAPDH, glyceraldehyde 3-phosphate dehydrogenase, PCCs, pancreatic cancer cells, PDAC, pancreatic ductal adenocarcinoma, STAT3, signal transducer and activator of transcription 3, TGF- $\beta$, transforming growth factor $-\beta$

\section{Declarations}

Ethics approval and consent to participate: The animal study was approved by the Institutional Animal Care and Use Committee of Wenzhou Medical University, China. Animal experiments were performed according to all regulatory and institutional guidelines for animal welfare (National Institutes of Health Publications, NIH 451 Publications No. 80-23).

Consent for publication: All authors agreed with submission of the manuscript for publication and agree to be accountable for all aspect of the manuscript.

Availability of data and materials: Not applicable.

Competing interests: The authors declare that they have no competing interest.

Funding: This study was sponsored by Key Laboratory of Diagnosis and Treatment of Severe HepatoPancreatic Diseases of Zhejiang Province (Grant No. 2018E10008).

Authors' contributions: GHC, ZQY and BYH conceived the study. GHC, XYY, YXJ, YZW, XLL, and CQB carried out the experiments. CJW, CCY, WMS, and SLM revised the statistical analyses. GHC and BYH drafted the manuscript. All authors contribute to and approved the final version of the manuscript.

Acknowledgements: Not applicable.

Authors' information: ${ }^{1}$ Key Laboratory of Diagnosis and Treatment of Severe Hepato-Pancreatic Diseases of Zhejiang Province, The First Affiliated Hospital of Wenzhou Medical University, Wenzhou 325000, China. ${ }^{2}$ Department of Hepato-Biliary-Pancreatic Surgery, The First Affiliated Hospital of Wenzhou Medical University, Wenzhou 325000, China. ${ }^{3}$ Health Assessment Center, Wenzhou Medical University, Wenzhou, 325000, China

\section{References}


1. Yoo W, Lee J, Jun E, Noh KH, Lee S, Jung D, Jung KH, Kim JS, Park YY, Kim SC et al: The YAP1-NMU Axis Is Associated with Pancreatic Cancer Progression and Poor Outcome: Identification of a Novel Diagnostic Biomarker and Therapeutic Target. Cancers (Basel) 2019, 11(10):1477.

2. Melisi D, Garcia-Carbonero R, Macarulla T, Pezet D, Deplanque G, Fuchs M, Trojan J, Oettle H, Kozloff $\mathrm{M}$, Cleverly A et al: Galunisertib plus gemcitabine vs. gemcitabine for first-line treatment of patients with unresectable pancreatic cancer. Br J Cancer 2018, 119(10):1208-1214.

3. Murphy JE, Wo JY, Ryan DP, Jiang W, Yeap BY, Drapek LC, Blaszkowsky LS, Kwak EL, Allen JN, Clark JW et al: Total Neoadjuvant Therapy With FOLFIRINOX Followed by Individualized Chemoradiotherapy for Borderline Resectable Pancreatic Adenocarcinoma: A Phase 2 Clinical Trial. JAMA Oncol 2018, 4(7):963-969.

4. Tu M, Li H, Lv N, Xi C, Lu Z, Wei J, Chen J, Guo F, Jiang K, Song G et al: Vasohibin 2 reduces chemosensitivity to gemcitabine in pancreatic cancer cells via Jun proto-oncogene dependent transactivation of ribonucleotide reductase regulatory subunit M2. Mol Cancer 2017, 16(1):66.

5. Kim JH, Lee J, Cho YR, Lee SY, Sung GJ, Shin DM, Choi KC, Son J: TFEB Supports Pancreatic Cancer Growth through the Transcriptional Regulation of Glutaminase. Cancers (Basel) 2021, 13(3):483.

6. Weng CC, Hsieh MJ, Wu CC, Lin YC, Shan YS, Hung WC, Chen LT, Cheng KH: Loss of the transcriptional repressor TGIF1 results in enhanced Kras-driven development of pancreatic cancer. Mol Cancer 2019, 18(1):96.

7. Wu SG, Shih JY: Management of acquired resistance to EGFR TKI-targeted therapy in advanced nonsmall cell lung cancer. Mol Cancer 2018, 17(1):38.

8. Jain P, Dvorkin-Gheva A, Mollen E, Malbeteau L, Xie M, Jessa F, Dhavarasa P, Chung S, Brown KR, Jang GH et al: NOX4 links metabolic regulation in pancreatic cancer to endoplasmic reticulum redox vulnerability and dependence on PRDX4. Sci Adv 2021, 7(19):eabf7114.

9. Wang X, Hu LP, Qin WT, Yang Q, Chen DY, Li Q, Zhou KX, Huang PQ, Xu CJ, Li J et al: Identification of a subset of immunosuppressive P2RX1-negative neutrophils in pancreatic cancer liver metastasis. Nat Commun 2021, 12(1):174.

10. Chien W, Sun QY, Lee KL, Ding LW, Wuensche P, Torres-Fernandez LA, Tan SZ, Tokatly I, Zaiden N, Poellinger $L$ et al: Activation of protein phosphatase $2 A$ tumor suppressor as potential treatment of pancreatic cancer. Mol Oncol 2015, 9(4):889-905.

11. Wang X, Xu Z, Sun J, Lv H, Wang Y, Ni Y, Chen S, Hu C, Wang L, Chen W et al: Cisplatin resistance in gastric cancer cells is involved with GPR30-mediated epithelial-mesenchymal transition. $J$ Cell Mol Med 2020, 24(6):3625-3633.

12. Liarte S, Bernabé-García Á, Nicolás FJ: Human Skin Keratinocytes on Sustained TGF- $\beta$ Stimulation Reveal Partial EMT Features and Weaken Growth Arrest Responses. Cells 2020, 9(1):255.

13. Liot S, Balas J, Aubert A, Prigent L, Mercier-Gouy P, Verrier B, Bertolino P, Hennino A, Valcourt U, Lambert E: Stroma Involvement in Pancreatic Ductal Adenocarcinoma: An Overview Focusing on Extracellular Matrix Proteins. Front Immuno/ 2021, 12:612271. 
14. Shields DJ, Murphy EA, Desgrosellier JS, Mielgo A, Lau SK, Barnes LA, Lesperance J, Huang M, Schmedt C, Tarin D et al: Oncogenic Ras/Src cooperativity in pancreatic neoplasia. Oncogene 2011, 30(18):2123-2134.

15. Teng M, Zhou S, Cai C, Lupien M, He HH: Pioneer of prostate cancer: past, present and the future of FOXA1. Protein Cell 2021, 12(1):29-38.

16. Kim J, Kang W, Kang SH, Park SH, Kim JY, Yang S, Ha SY, Paik YH: Proline-rich tyrosine kinase 2 mediates transforming growth factor-beta-induced hepatic stellate cell activation and liver fibrosis. Sci Rep 2020, 10(1):21018.

17. Lankadasari MB, Aparna JS, Mohammed S, James S, Aoki K, Binu VS, Nair S, Harikumar KB: Targeting S1PR1/STAT3 loop abrogates desmoplasia and chemosensitizes pancreatic cancer to gemcitabine. Theranostics 2018, 8(14):3824-3840.

18. Lee JH, Mohan CD, Deivasigamani A, Jung YY, Rangappa S, Basappa S, Chinnathambi A, Alahmadi TA, Alharbi SA, Garg M et al: Brusatol suppresses STAT3-driven metastasis by downregulating epithelial-mesenchymal transition in hepatocellular carcinoma. $J$ Adv Res 2020, 26:83-94.

19. Laklai H, Miroshnikova YA, Pickup MW, Collisson EA, Kim GE, Barrett AS, Hill RC, Lakins JN, Schlaepfer DD, Mouw JK et al: Genotype tunes pancreatic ductal adenocarcinoma tissue tension to induce matricellular fibrosis and tumor progression. Nat Med 2016, 22(5):497-505.

20. Drubay V, Skrypek N, Cordiez L, Vasseur R, Schulz C, Boukrout N, Duchêne B, Coppin L, Van Seuningen I, Jonckheere N: TGF- $\beta$ RII Knock-down in Pancreatic Cancer Cells Promotes Tumor Growth and Gemcitabine Resistance. Importance of STAT3 Phosphorylation on S727. Cancers (Basel) 2018, 10(8):254.

21. Zhao S, Venkatasubbarao K, Lazor JW, Sperry J, Jin C, Cao L, Freeman JW: Inhibition of STAT3 Tyr705 phosphorylation by Smad4 suppresses transforming growth factor beta-mediated invasion and metastasis in pancreatic cancer cells. Cancer Res 2008, 68(11):4221-4228.

22. Guo Y, Tong Y, Zhu H, Xiao Y, Guo H, Shang L, Zheng W, Ma S, Liu X, Bai Y: Quercetin suppresses pancreatic ductal adenocarcinoma progression via inhibition of SHH and TGF- $\beta /$ Smad signaling pathways. Cell Biol Toxicol 2021, 37(3):479-496.

23. Huang C, Li Y, Li Z, Xu Y, Li N, Ge Y, Dong J, Chang A, Zhao T, Wang X et al: LIMS1 Promotes Pancreatic Cancer Cell Survival under Oxygen-Glucose Deprivation Conditions by Enhancing HIF1A Protein Translation. Clin Cancer Res 2019, 25(13):4091-4103.

24. Tang Q, Chen J, Di Z, Yuan W, Zhou Z, Liu Z, Han S, Liu Y, Ying G, Shu X et al: TM4SF1 promotes EMT and cancer stemness via the Wnt/ $\beta$-catenin/SOX2 pathway in colorectal cancer. J Exp Clin Cancer Res 2020, 39(1):232.

25. Zhang X, Lu H, Xie S, Wu C, Guo Y, Xiao Y, Zheng S, Zhu H, Zhang Y, Bai Y: Resveratrol suppresses the myofibroblastic phenotype and fibrosis formation in kidneys via proliferation-related signalling pathways. Br J Pharmaco/2019, 176(24):4745-4759.

26. Zheng X, Carstens JL, Kim J, Scheible M, Kaye J, Sugimoto H, Wu CC, LeBleu VS, Kalluri R: Epithelialto-mesenchymal transition is dispensable for metastasis but induces chemoresistance in pancreatic 
cancer. Nature 2015, 527(7579):525-530.

27. Mittal V: Epithelial Mesenchymal Transition in Tumor Metastasis. Annu Rev Pathol 2018, 13:395412.

28. Nagel G, Sousa-Herves A, Wedepohl S, Calderón M: Matrix Metalloproteinase-sensitive Multistage Nanogels Promote Drug Transport in 3D Tumor Model. Theranostics 2020, 10(1):91-108.

29. Rajendran P, Ben Ammar R, Al-Saeedi FJ, Elsayed Mohamed M, Islam M, Al-Ramadan SY: Thidiazuron decreases epithelial-mesenchymal transition activity through the NF-kB and PI3K/AKT signalling pathways in breast cancer. J Cell Mol Med 2020, 24(24):14525-14538.

30. Xu Y, Chang R, Peng Z, Wang Y, Ji W, Guo J, Song L, Dai C, Wei W, Wu Y et al: Loss of polarity protein AF6 promotes pancreatic cancer metastasis by inducing Snail expression. Nat Commun 2015, 6:7184.

31. Azoitei N, Becher A, Steinestel K, Rouhi A, Diepold K, Genze F, Simmet T, Seufferlein T: PKM2 promotes tumor angiogenesis by regulating HIF-1a through NF-kB activation. Mol Cancer 2016, 15:3.

32. He L, Zhu W, Chen Q, Yuan Y, Wang Y, Wang J, Wu X: Ovarian cancer cell-secreted exosomal miR-205 promotes metastasis by inducing angiogenesis. Theranostics 2019, 9(26):8206-8220.

33. Ciccone V, Terzuoli E, Donnini S, Giachetti A, Morbidelli L, Ziche M: Stemness marker ALDH1A1 promotes tumor angiogenesis via retinoic acid/HIF-1a/VEGF signalling in MCF-7 breast cancer cells. J Exp Clin Cancer Res 2018, 37(1):311.

34. David CJ, Huang YH, Chen M, Su J, Zou Y, Bardeesy N, lacobuzio-Donahue CA, Massagué J: TGF- $\boldsymbol{\beta}$ Tumor Suppression through a Lethal EMT. Cell 2016, 164(5):1015-1030.

35. Kim JY, An HJ, Kim WH, Gwon MG, Gu H, Park YY, Park KK: Anti-fibrotic Effects of Synthetic Oligodeoxynucleotide for TGF- $\beta 1$ and Smad in an Animal Model of Liver Cirrhosis. Mol Ther Nucleic Acids 2017, 8:250-263.

36. Miguel V, Ramos R, García-Bermejo L, Rodríguez-Puyol D, Lamas S: The program of renal fibrogenesis is controlled by microRNAs regulating oxidative metabolism. Redox Bio/ 2021, 40:101851.

37. McMurray JS: A new small-molecule Stat3 inhibitor. Chem Biol 2006, 13(11):1123-1124.

38. Liang C, Xu J, Meng Q, Zhang B, Liu J, Hua J, Zhang Y, Shi S, Yu X: TGFB1-induced autophagy affects the pattern of pancreatic cancer progression in distinct ways depending on SMAD4 status. Autophagy 2020, 16(3):486-500.

39. Zhang X, Lu H, Hong W, Liu L, Wang S, Zhou M, Chen B, Bai Y: Tyrphostin B42 attenuates trichostatin A-mediated resistance in pancreatic cancer cells by antagonizing IL-6/JAK2/STAT3 signaling. Oncol Rep 2018, 39(4):1892-1900.

40. Wang G, Yu Y, Sun C, Liu T, Liang T, Zhan L, Lin X, Feng XH: STAT3 selectively interacts with Smad3 to antagonize TGF- $\beta$ signalling. Oncogene 2016, 35(33):4388-4398.

41. Zhang LN, Kong CF, Zhao D, Cong XL, Wang SS, Ma L, Huang YH: Fusion with mesenchymal stem cells differentially affects tumorigenic and metastatic abilities of lung cancer cells. J Cell Physiol 
2019, 234(4):3570-3582.

42. Lee S, Goldfinger LE: RLIP76 regulates HIF-1 activity, VEGF expression and secretion in tumor cells, and secretome transactivation of endothelial cells. FASEB J 2014, 28(9):4158-4168.

43. Xia Y, Wang G, Jiang M, Liu X, Zhao Y, Song Y, Jiang B, Zhu D, Hu L, Zhang Z et al: A Novel Biological Activity of the STAT3 Inhibitor Stattic in Inhibiting Glutathione Reductase and Suppressing the Tumorigenicity of Human Cervical Cancer Cells via a ROS-Dependent Pathway. Onco Targets Ther $2021,14: 4047-4060$.

44. Khaki-Khatibi F, Ghorbani M, Sabzichi M, Ramezani F, Mohammadian J: Adjuvant therapy with stattic enriches the anti-proliferative effect of doxorubicin in human ZR-75-1 breast cancer cells via arresting cell cycle and inducing apoptosis. Biomed Pharmacother 2019, 109:1240-1248.

45. Thulin MH, Määttä J, Linder A, Sterbova S, Ohlsson C, Damber JE, Widmark A, Persson E: Inhibition of STAT3 prevents bone metastatic progression of prostate cancer in vivo. Prostate 2021, 81(8):452462.

46. Lin L, Jou D, Wang Y, Ma H, Liu T, Fuchs J, Li PK, Lü J, Li C, Lin J: STAT3 as a potential therapeutic target in ALDH+ and CD44+/CD24+ stem cell-like pancreatic cancer cells. Int J Oncol 2016, 49(6):2265-2274.

47. Su J, Morgani SM, David CJ, Wang Q, Er EE, Huang YH, Basnet H, Zou Y, Shu W, Soni RK et al: TGF- $\beta$ orchestrates fibrogenic and developmental EMTs via the RAS effector RREB1. Nature 2020, 577(7791):566-571.

48. Liu YY, Zhang S, Yu TJ, Zhang FL, Yang F, Huang YN, Ma D, Liu GY, Shao ZM, Li DQ: Pregnancyspecific glycoprotein 9 acts as both a transcriptional target and a regulator of the canonical TGF$\beta /$ Smad signaling to drive breast cancer progression. Clin Trans/ Med 2020, 10(8):e245.

49. Xu M, He J, Li J, Feng W, Zhou H, Wei H, Zhou M, Lu Y, Zeng J, Peng W et al: Methyl-CpG-binding domain 3 inhibits epithelial-mesenchymal transition in pancreatic cancer cells via TGF- $\beta / \mathrm{Smad}$ signalling. Br J Cancer 2017, 116(1):91-99.

50. Zhong M, Huang Z, Wang L, Lin Z, Cao Z, Li X, Zhang F, Wang H, Li Y, Ma X: Malignant Transformation of Human Bronchial Epithelial Cells Induced by Arsenic through STAT3/miR301a/SMAD4 Loop. Sci Rep 2018, 8(1):13291.

51. Junk DJ, Bryson BL, Smigiel JM, Parameswaran N, Bartel CA, Jackson MW: Oncostatin M promotes cancer cell plasticity through cooperative STAT3-SMAD3 signaling. Oncogene 2017, 36(28):40014013.

52. Ruoming $\mathrm{W}$, Zhen $\mathrm{Y}$, Tengteng $\mathrm{Z}$, Jisheng $\mathrm{H}$ : Tumor suppressor microRNA-31 inhibits gastric carcinogenesis by targeting Smad4 and SGPP2. Cancer Gene Ther 2015, 22(12):564-572.

\section{Figures}




\section{Figure 1}

Stattic inhibits PCC invasion and infiltration. A 2D structure and 3D conformation of Stattic. B 0, 24, and $48 \mathrm{~h}$ scratch wound healing assays after Stattic $(0,4$, and $10 \mu \mathrm{M})$ treatment of PANC- 1 cells. C The statistical results of scratch wound healing assays show that Stattic can significantly inhibit PANC-1 cell migration. D 0, 24, and $48 \mathrm{~h}$ wound healing assays after Stattic (0, 4, and $10 \mu \mathrm{M})$ treatment of BxPc-3 cells. E The statistical results of scratch wound healing assays show that Stattic can significantly inhibit BxPc-3 cell migration. F Transwell chamber assays of PANC- 1 and BxPc-3 cells treated with Stattic $(0,4$, and $10 \mu \mathrm{M})$. G The statistical results of Transwell chamber assays show that Stattic can significantly inhibit the invasion of PANC-1 cells. $\mathrm{H}$ The statistical results of Transwell chamber assays show that Stattic can significantly inhibit the invasiveness of BxPc-3 cells. The experiment was repeated in independent triplicates. Results are expressed as the mean $\pm S D$. ${ }^{\star} P<0.05,{ }^{*} P<0.01$, and ${ }^{* \star * P}<0.001$. 
A
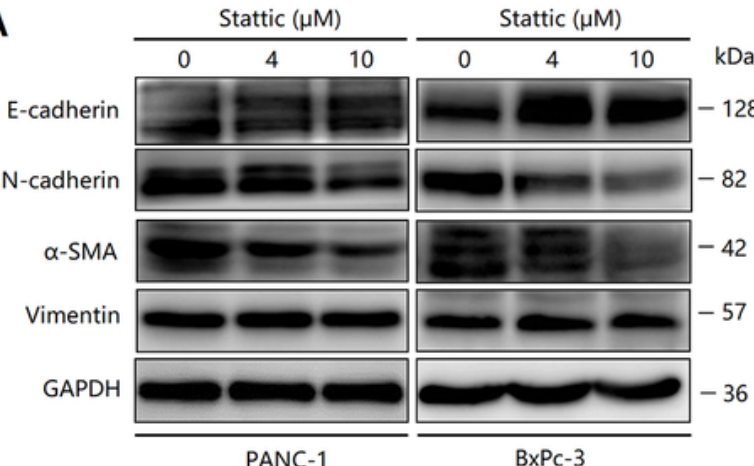

B
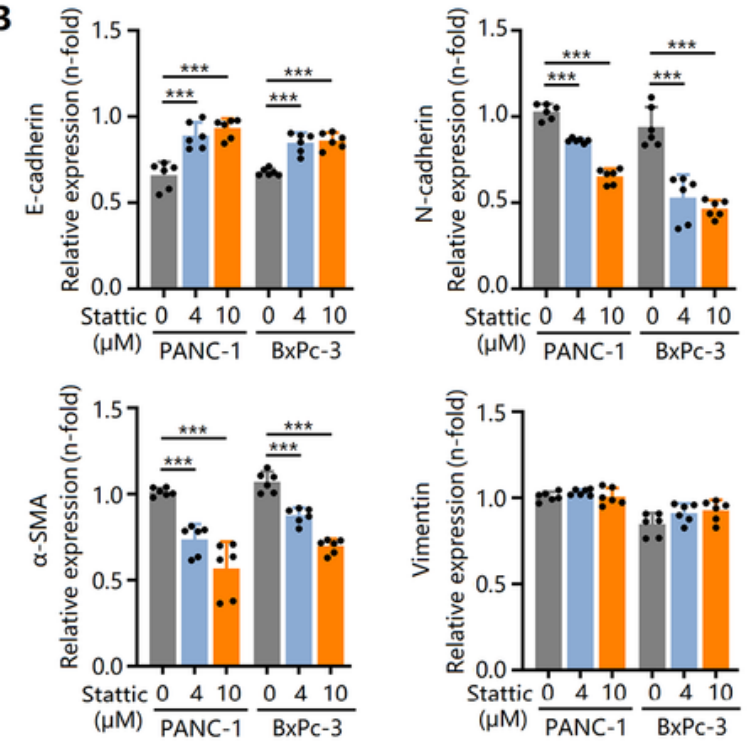

D

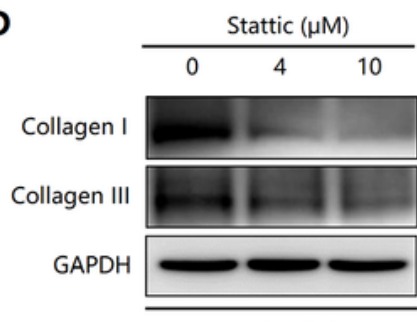

PANC-1

E

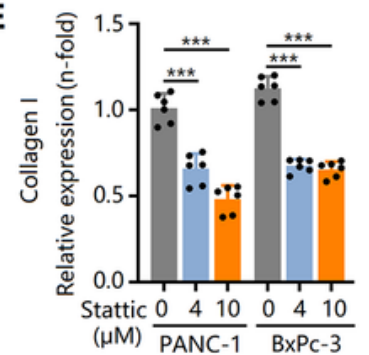

BxPc-3
C
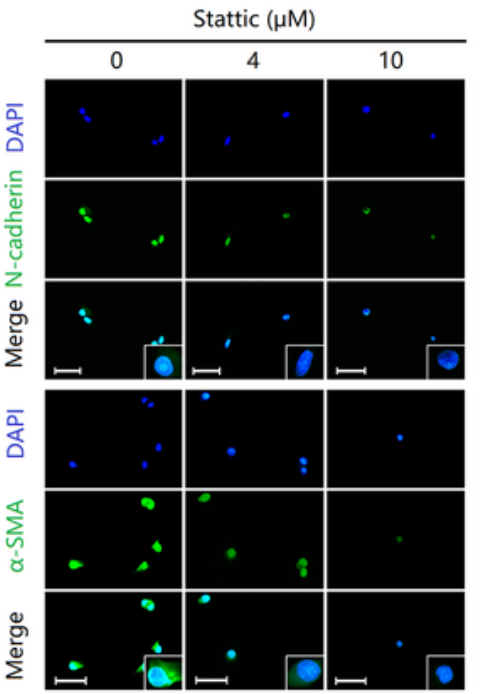

PANC-1

$\mathbf{F}$

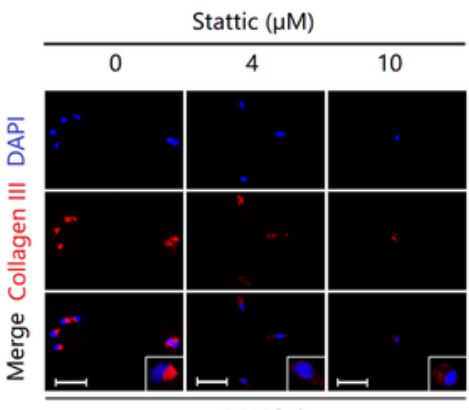

PANC-1

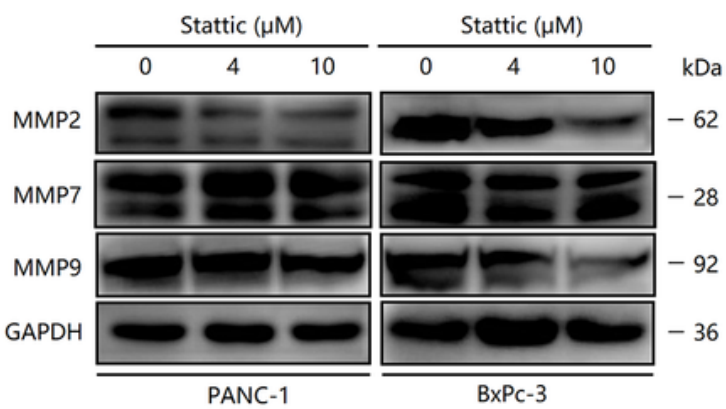

${ }^{H}$

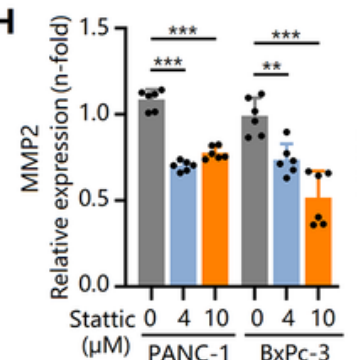

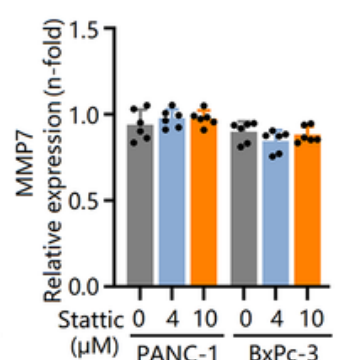

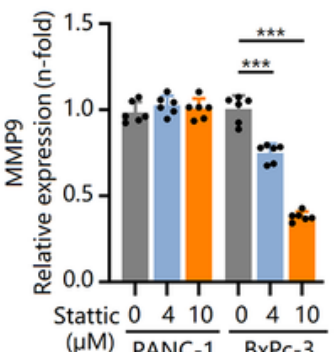

Figure 2

Stattic antagonizes EMT in pancreatic cancer cells. A E-cadherin, N-cadherin, a-SMA, and vimentin western blot results of PANC-1 and BxPc-3 cells after Stattic $(0,4$, and $10 \mu \mathrm{M})$ treatment. B E-cadherin, Ncadherin, a-SMA, and vimentin western blot statistical results of PANC-1 and BxPc-3 cells after Stattic (0, 4, and $10 \mu \mathrm{M}$ ) treatment. C IF staining of N-cadherin (green) and a-SMA (green) in PANC-1 and BxPc-3 cells with or without Stattic treatment. The nucleus was counterstained with DAPI (blue), bar = $25 \mu \mathrm{m}$. D 
collagen I and collagen III western blot results of PANC-1 and BxPc-3 cells after Stattic $(0,4$, and $10 \mu \mathrm{M})$ treatment. E The statistical results of western blot show that Stattic treatment downregulated the protein expression levels of collagen I and collagen III. F IF staining of collagen III (red) in PANC-1 and BxPc-3 cells with or without Stattic treatment. The nucleus was counterstained with DAPI (blue), bar $=25 \mu \mathrm{m}$. G MMP2, MMP7, and MMP9 western blot results of PANC-1 and BxPc-3 cells after Stattic $(0,4$, and $10 \mu \mathrm{M})$ treatment. H MMP2, MMP7, and MMP9 western blot statistical results of PANC-1 and BxPc-3 cells after Stattic $(0,4$, and $10 \mu \mathrm{M})$ treatment. The experiment was repeated in independent triplicates. Results are expressed as the mean $\pm \mathrm{SD}$. ${ }^{*} \mathrm{P}<0.01$ and ${ }^{* *} \mathrm{P}<0.001$. 
A

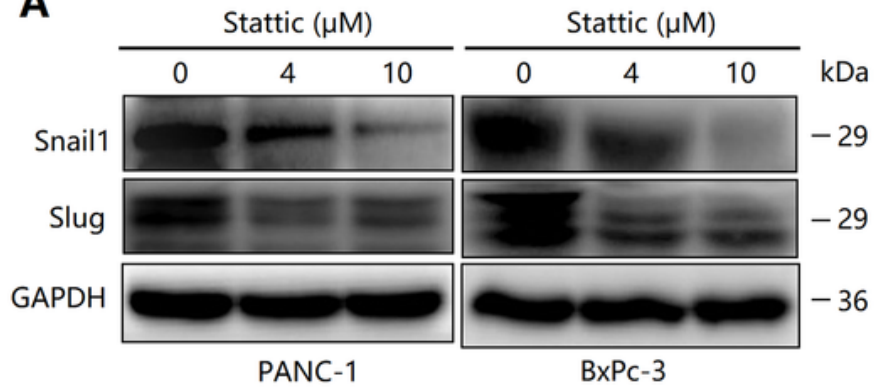

C

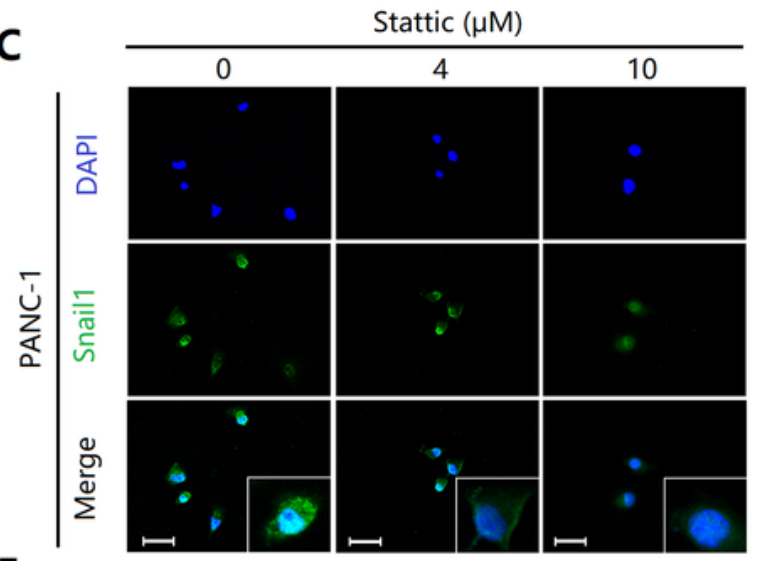

E

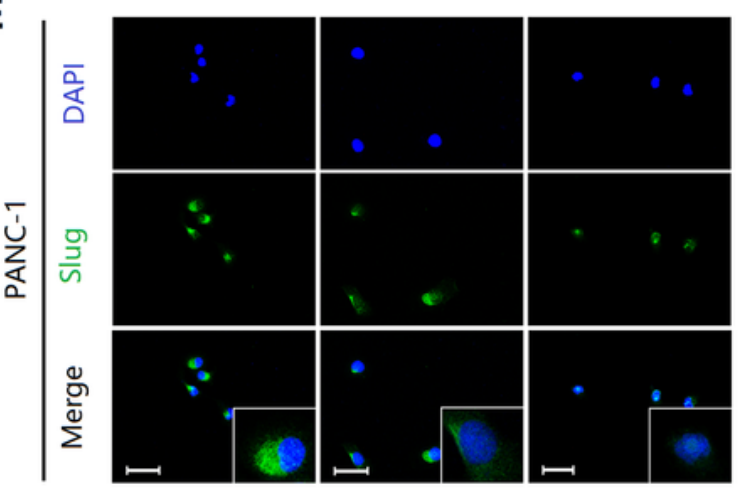

G
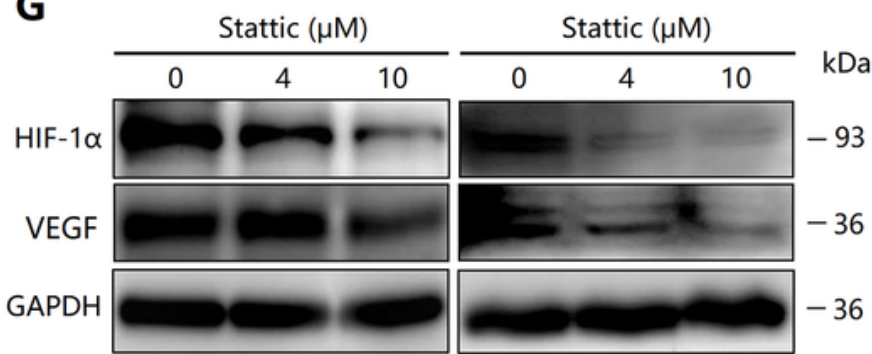

PANC-1

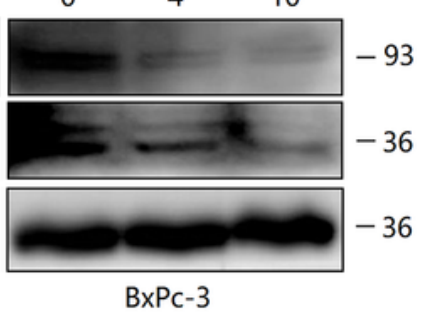

BxPc-3
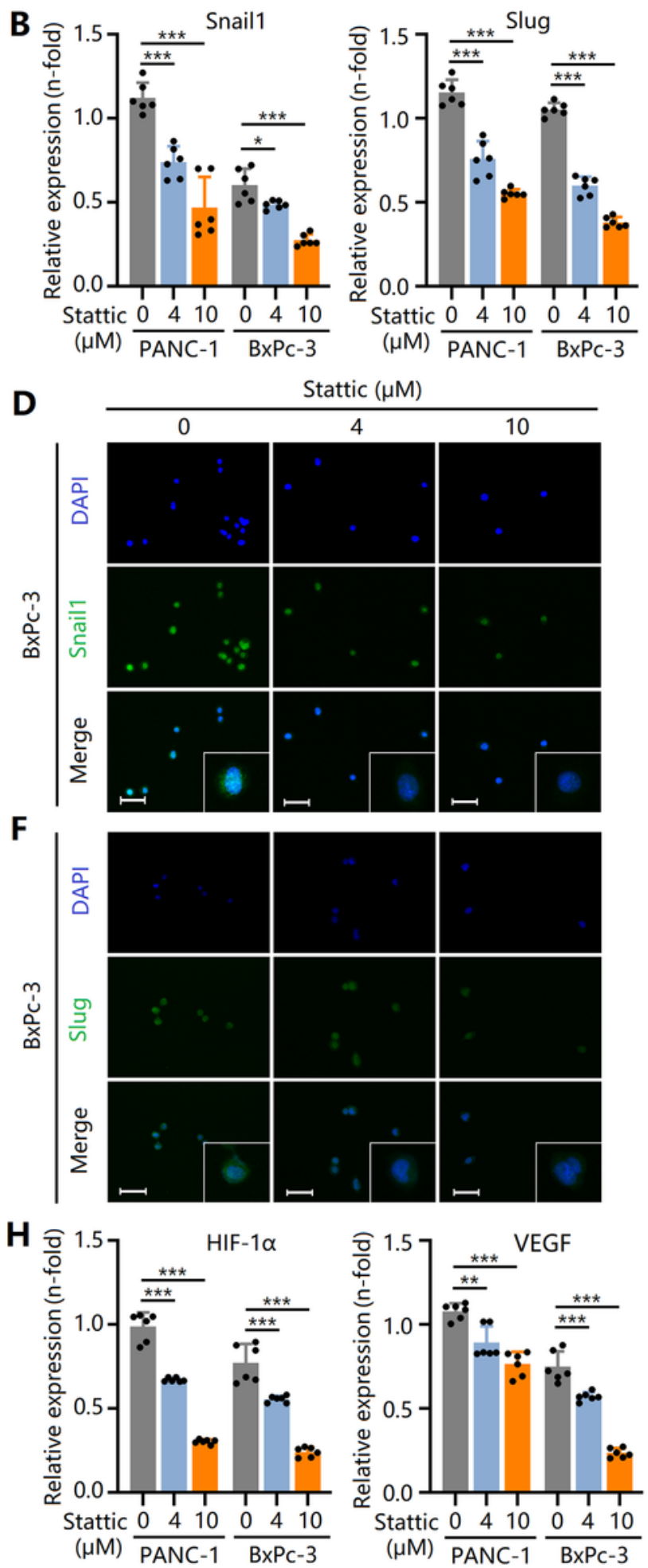

\section{Figure 3}

Stattic inhibits EMT-TF and VEGF expression in pancreatic cancer cells. A Snail1 and Slug western blot results of PANC- 1 and BxPc-3 cells after Stattic $(0,4$, and $10 \mu \mathrm{M})$ treatment. B The statistical results of western blot show that Stattic treatment downregulated the protein expression levels of Snail1 and Slug. C-F IF staining of Snail1 (green) and Slug (green) in PANC-1 and BxPc-3 cells with or without Stattic treatment. The nucleus was counterstained with DAPI (blue), bar $=25 \mu \mathrm{m}$. G HIF-1a and VEGF western 
blot results of PANC-1 and BxPc-3 cells after Stattic $(0,4$, and $10 \mu \mathrm{M})$ treatment. $\mathrm{H}$ The statistical results of western blot show that Stattic treatment downregulated the protein expression levels of HIF-1 $\mathrm{a}$ and VEGF. The experiment was repeated in independent triplicates. Results are expressed as the mean \pm SD. ${ }^{*} \mathrm{P}<0.05, * * \mathrm{P}<0.01$, and ${ }^{* * *} \mathrm{P}<0.001$.
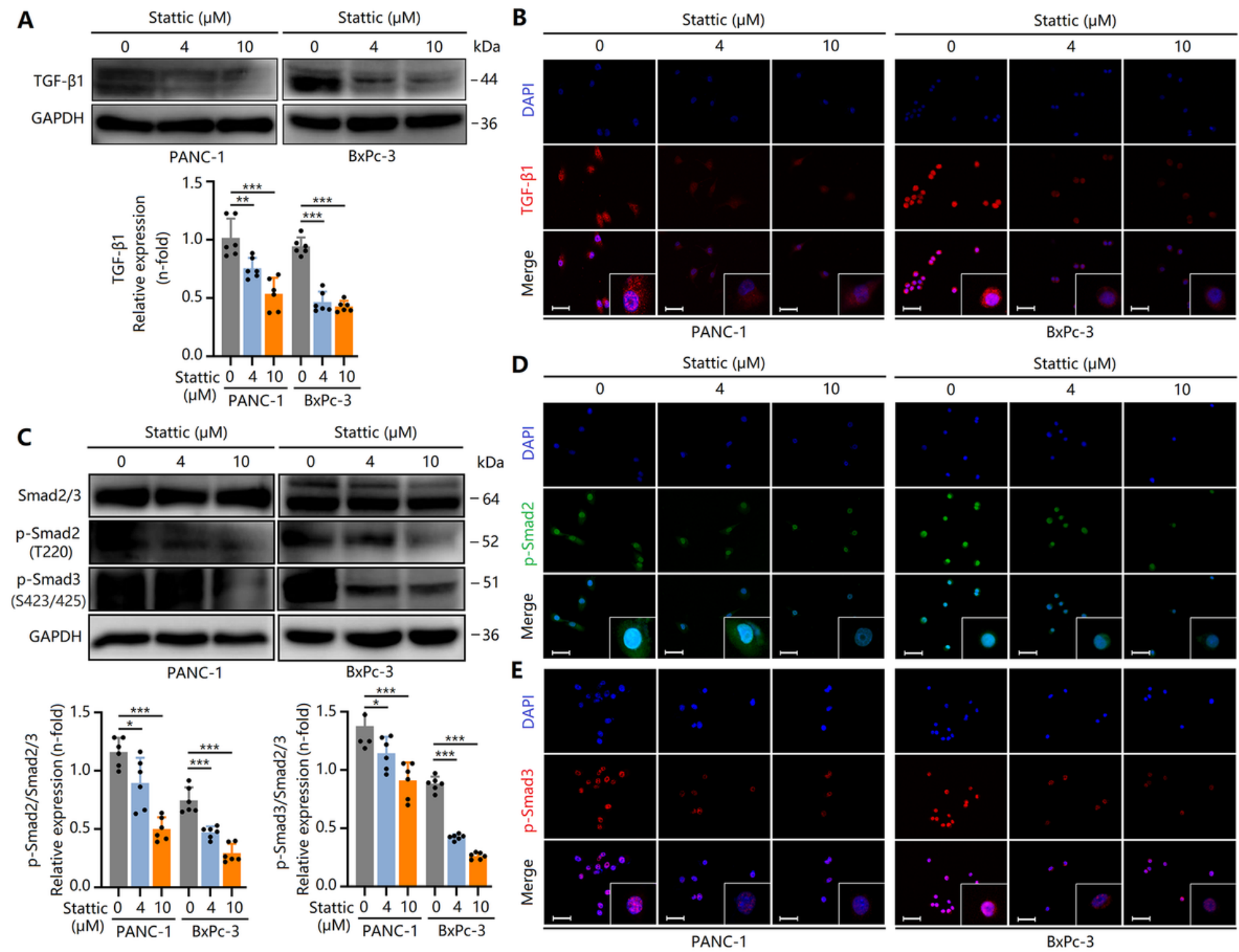

\section{Figure 4}

Stattic inhibits TGF/Smad signaling activation in pancreatic cancer cells. A The western blot results and statistical analysis of PANC-1 and BxPc-3 shows that Stattic (4 and $10 \mu \mathrm{M})$ treatment downregulate the protein expression of TGF- $\beta 1$. B IF staining of TGF- $\beta 1$ (red) in PANC-1 and BxPc-3 cells with or without Stattic treatment. The nucleus was counterstained with DAPI (blue), bar $=25 \mu \mathrm{m}$. C The western blot and statistical analysis of Smad2/3, p-Smad2, and p-Smad3 in PANC-1 and BxPc-3 cells with or without Stattic treatment. D, E IF staining of p-Smad2 (green) and p-Smad3 (red) in PANC-1 and BxPc-3 cells with or without Stattic treatment, the nucleus was counterstained with DAPI (blue), bar $=25 \mu \mathrm{m}$. The experiment was repeated in independent triplicates. Results are expressed as the mean $\pm S D$. ${ }^{*} P<0.05$, ${ }^{*} \mathrm{P}<0.01$, and ${ }^{* \star *} \mathrm{P}<0.001$. 
A

PANC-1 Cells $\left(5 \times 10^{6} / 100 \mu \mathrm{l}\right)$

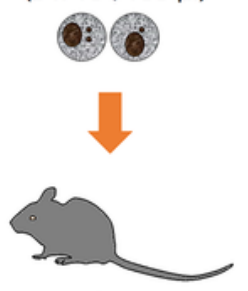

Nude mice

(Six in each group)

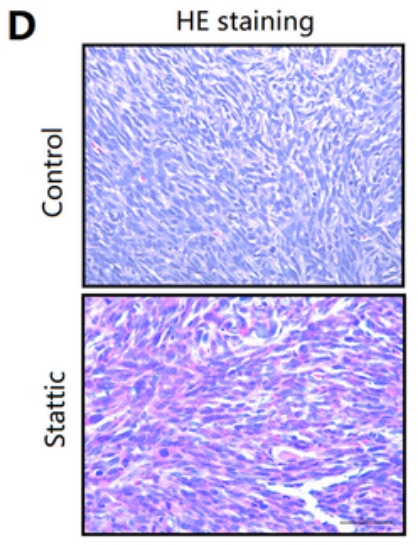

G

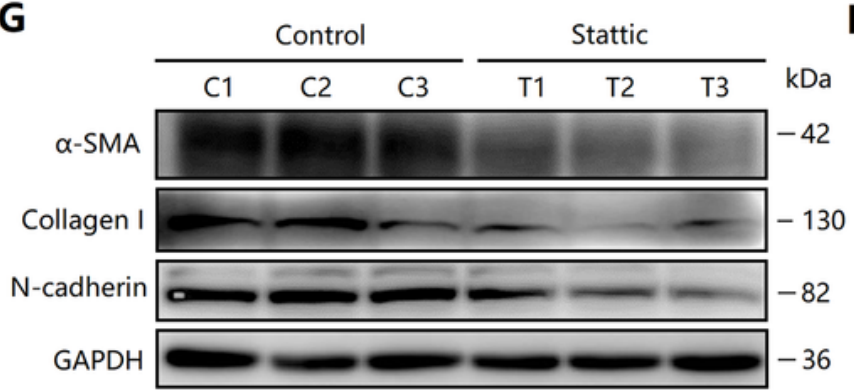

I
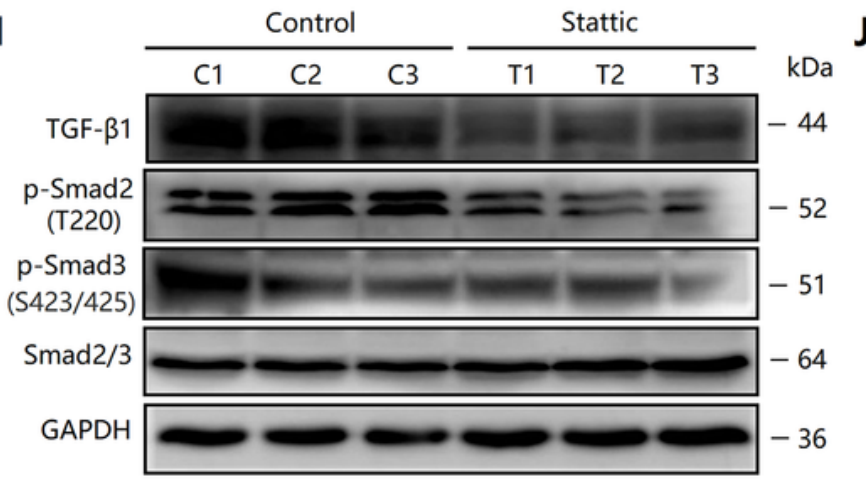

B

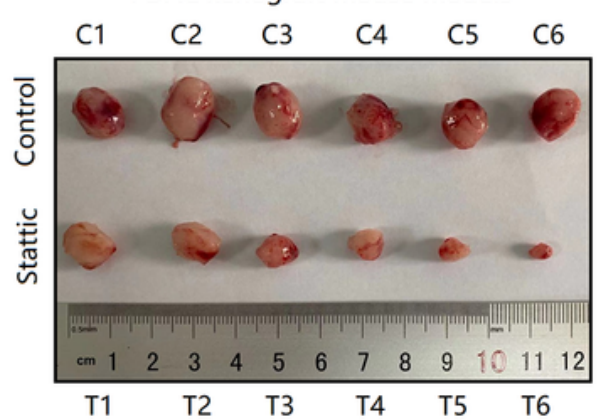

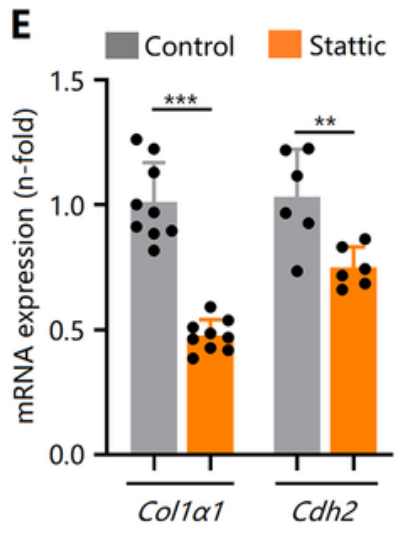

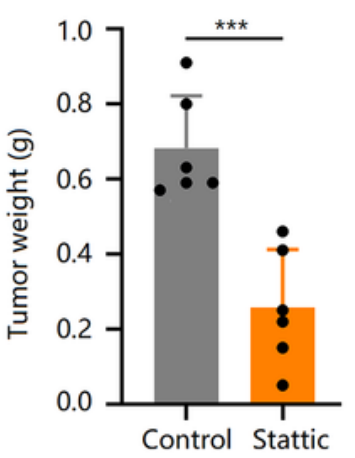

$\mathbf{F}$
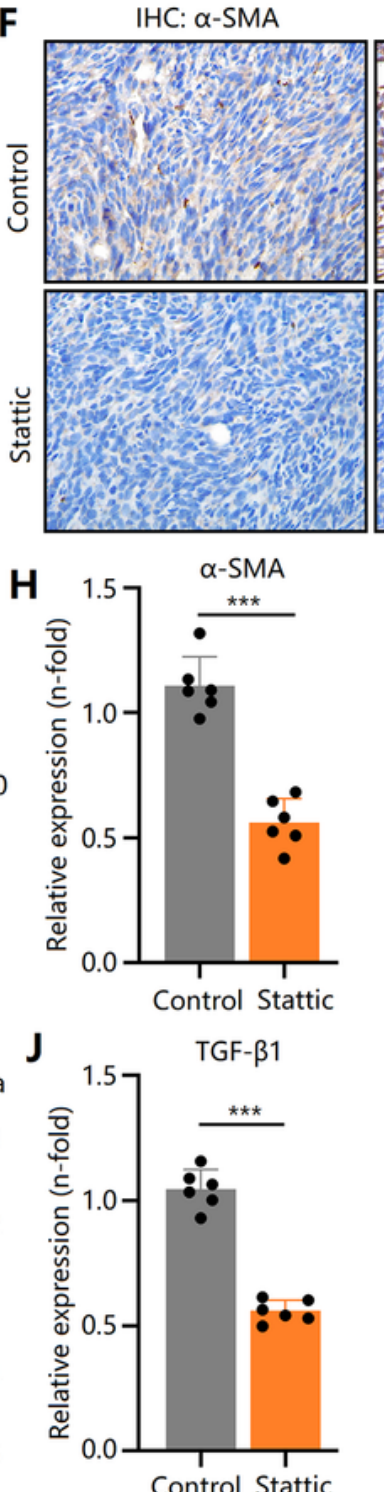

IHC: N-cadherin
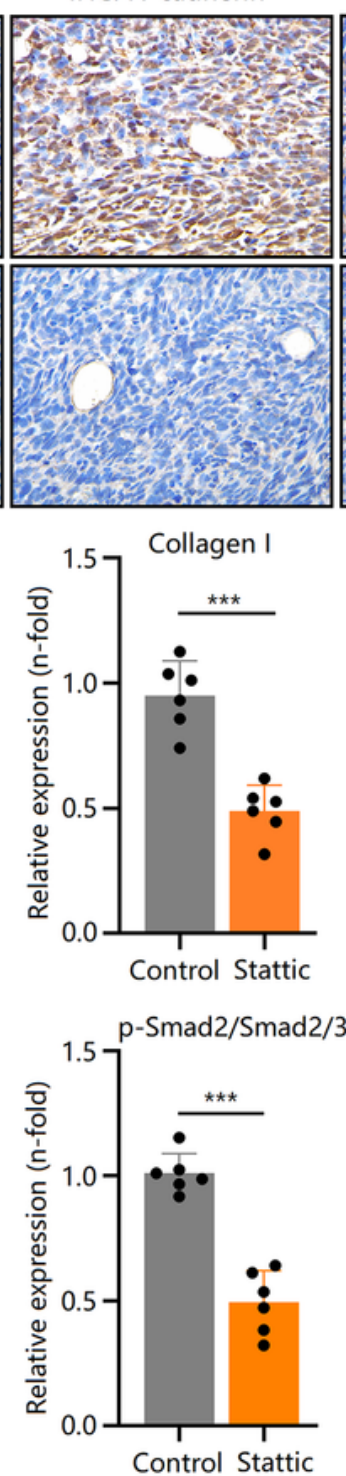

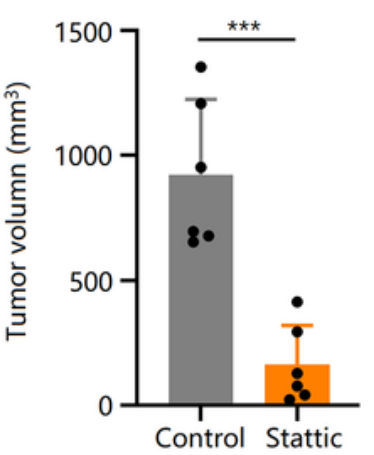

IHC: Collagen I
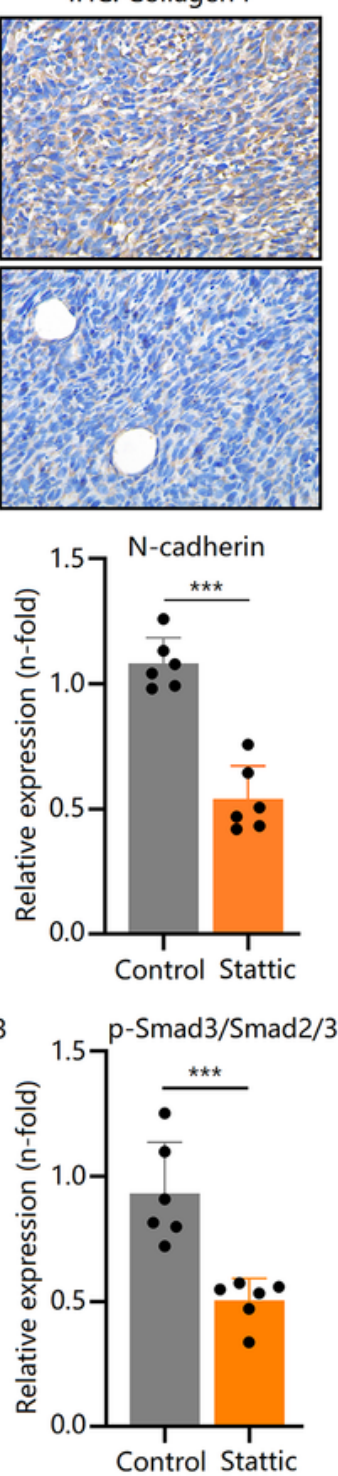

\section{Figure 5}

Stattic inhibits PDA growth and EMT. A Animal xenograft model construction and grouping and nude mouse tumor images from the Stattic treatment group and control group. B The nude mouse tumor weight in the Stattic treatment group was lower than that in the control group. C The nude mouse tumor volume in the Stattic treatment group was lower than that in the control group. D HE staining of nude mouse tumors in the Stattic and control groups. E mRNA expression of Col1a1 and Cdh2 in nude mouse 
tumors with or without Stattic treatment. F IHC staining of N-cadherin, a-SMA, and collagen nude mouse tumors with or without Stattic treatment. G a-SMA, collagen I, and N-cadherin western blot results in nude mouse tumors after Stattic treatment. H Western blot statistical results of a-SMA, collagen I, and Ncadherin with or without Stattic treatment. I TGF- $\beta 1$, p-Smad2, p-Smad3, and Smad2/3 western blot of nude mouse tumors after Stattic treatment. J Western blot statistical results of TGF- $\beta 1$, p-Smad2, and pSmad3 with or without Stattic treatment. The experiment was repeated in independent triplicates. Results are expressed as the mean $\pm \mathrm{SD}$. ${ }^{*} \mathrm{P}<0.01$ and ${ }^{*} * \mathrm{P}<0.001$.

A

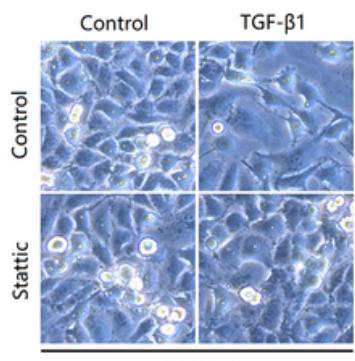

PANC-1

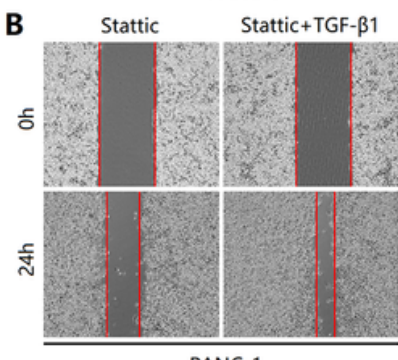

PANC-1
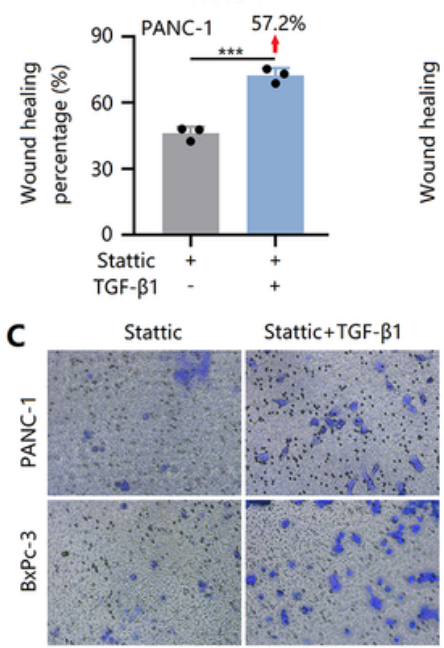

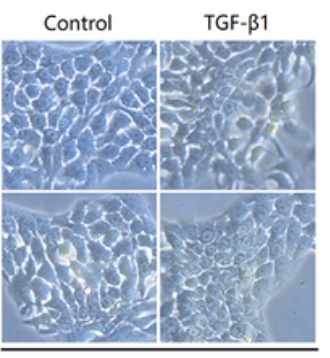

BxPc-3
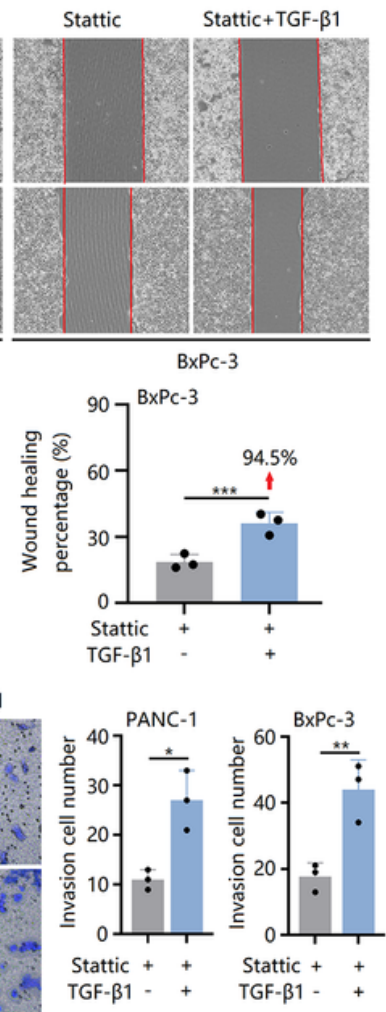

D

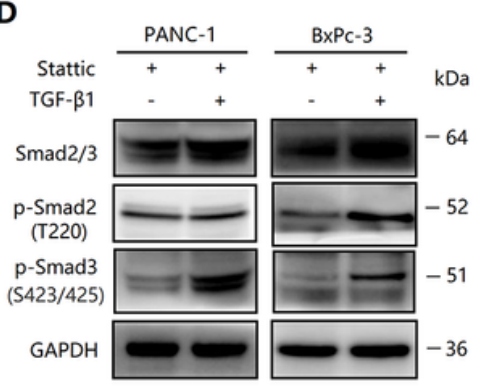

$\mathbf{F}$

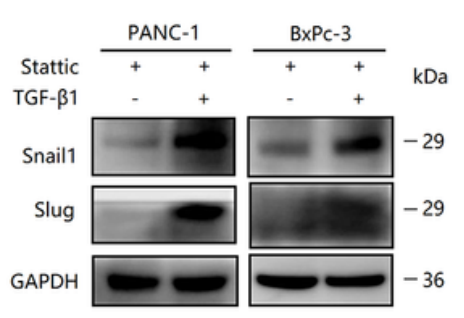

G

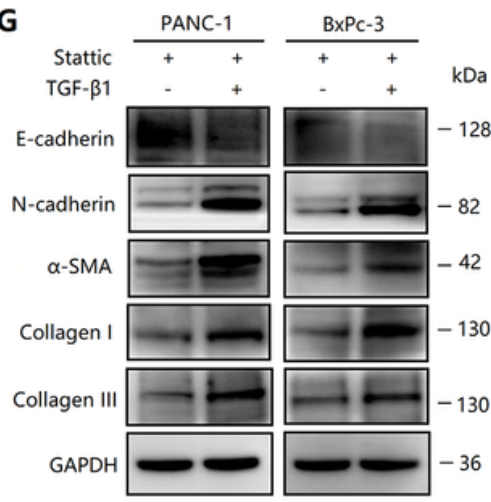

E

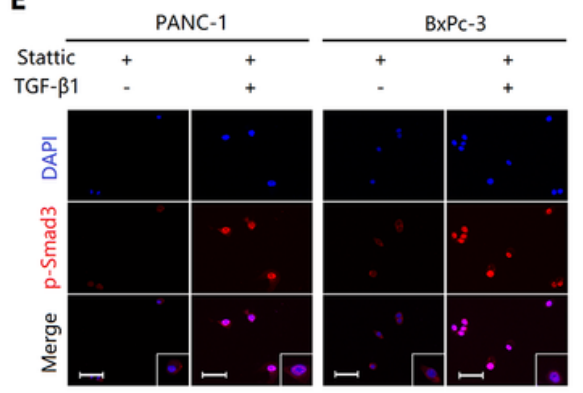

H

Stattic

TGF- $\beta 1$
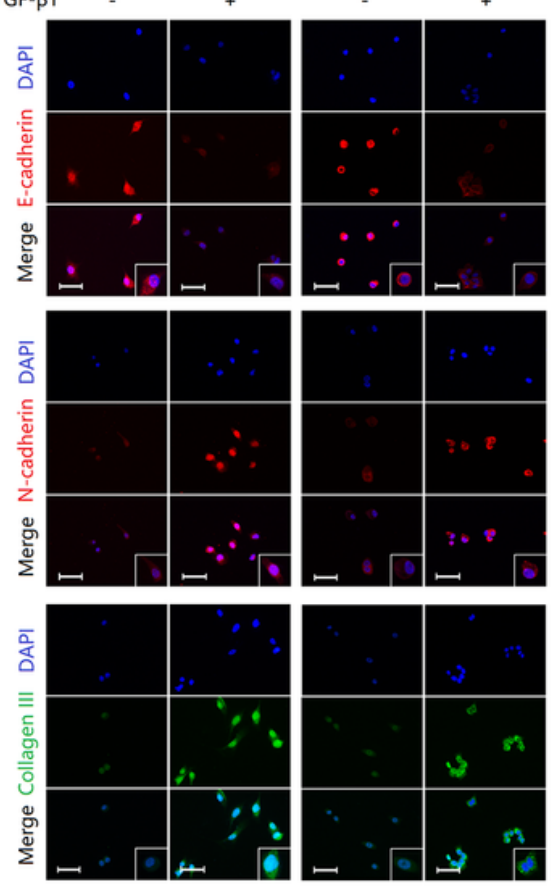

BxPc-3
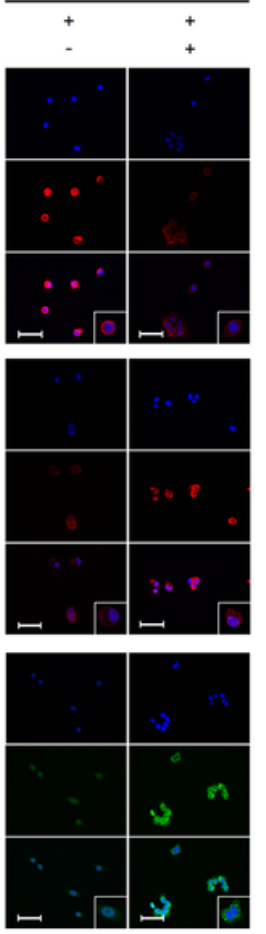

\section{Figure 6}

TGF- $\beta 1$ antagonizes the inhibitory effects of Stattic on pancreatic cancer cells. A Cell morphology images show that fibrosis increased in PANC-1 and BxPc-3 cells after TGF- $\beta 1$ treatment. B Images and statistical analysis of scratch wound healing assays show that TGF- $\beta 1$ antagonizes the inhibitory effects of Stattic on migration in PANC-1 and BxPc-3 cells. C Images and statistical analysis of Transwell chamber assays show that TGF- $\beta 1$ antagonizes the inhibitory effects of Stattic on invasiveness in PANC-1 and BxPc-3 cells. D Western blot results of Smad2/3, p-Smad2, and p-Smad3 protein expression in Stattic-treated PANC-1 and BxPc-3 cells with or without TGF- $\beta 1$. E IF staining of p-Smad3 (red) in Stattic-treated PANC-1 
and BxPc-3 cells with or without TGF- $\beta 1$, the nucleus was counterstained with DAPI (blue), bar $=25 \mu \mathrm{m}$. F Western blot results of Snail 1 and Slug protein expression in Stattic-treated PANC-1 and BxPc-3 cells with or without TGF- $\beta 1$. G Western blot results of EMT-related protein expression in Stattic-treated PANC-1 and BxPc-3 cells with or without TGF- $\beta 1$. H IF staining of E-cadherin (red), N-cadherin (red) and collagen III (green) in Stattic-treated PANC-1 and BxPc-3 cells with or without TGF- $\beta 1$, the nucleus was counterstained with DAPI (blue), bar $=25 \mu \mathrm{m}$. The experiment was repeated in independent triplicates. Results are expressed as the mean $\pm S D$. ${ }^{*} P<0.05, * * P<0.01$, and $* * * P<0.001$.

A

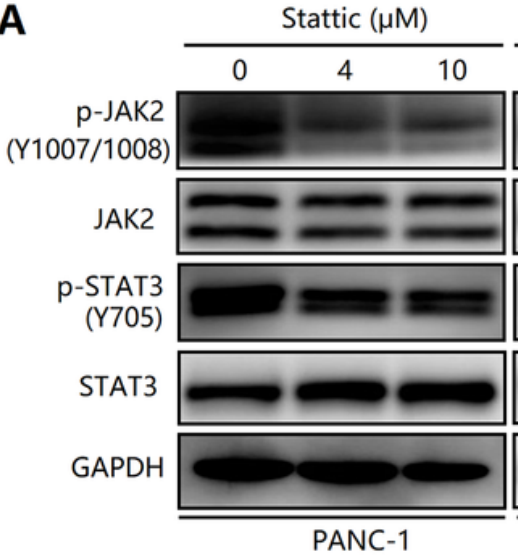

C

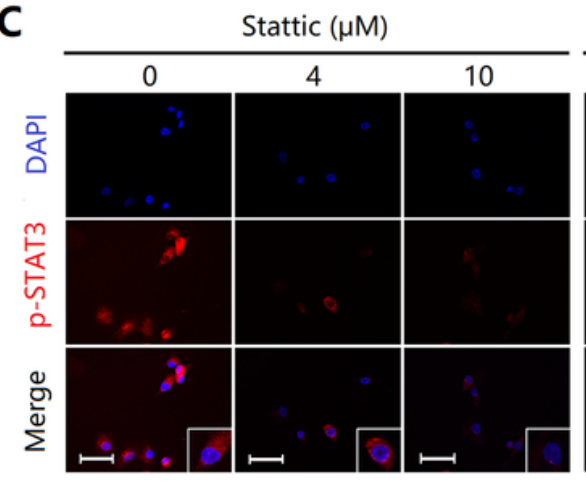

D

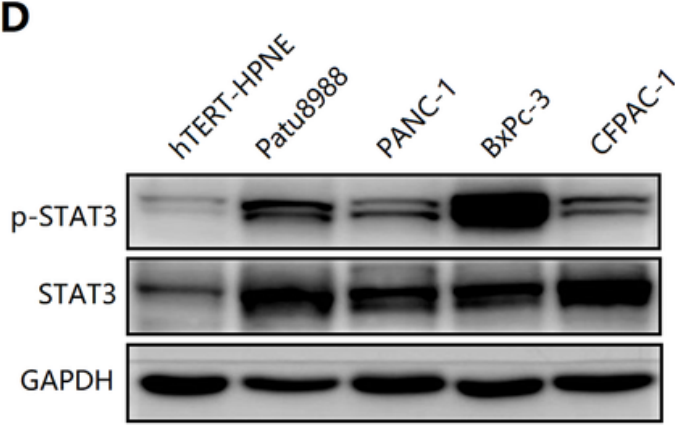

Stattic $(\mu \mathrm{M})$

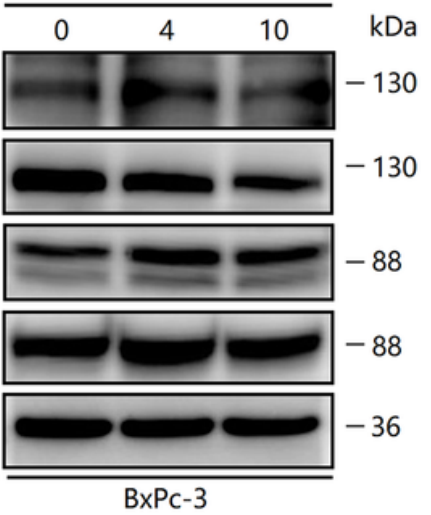

BxPc-3
B

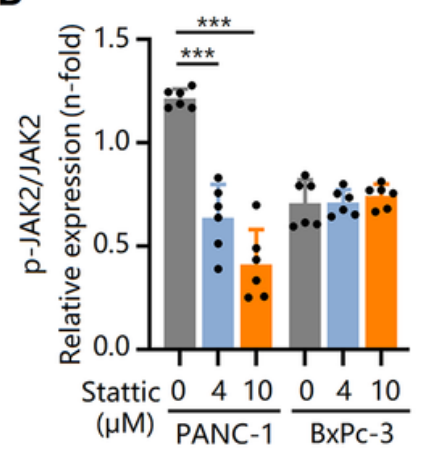

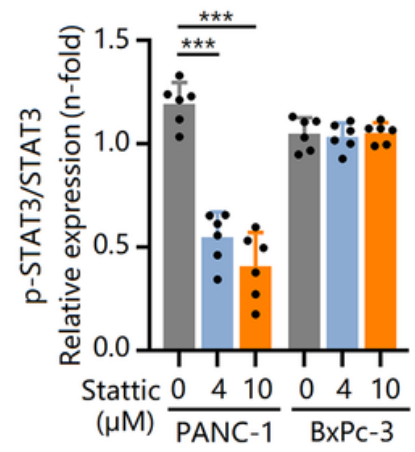

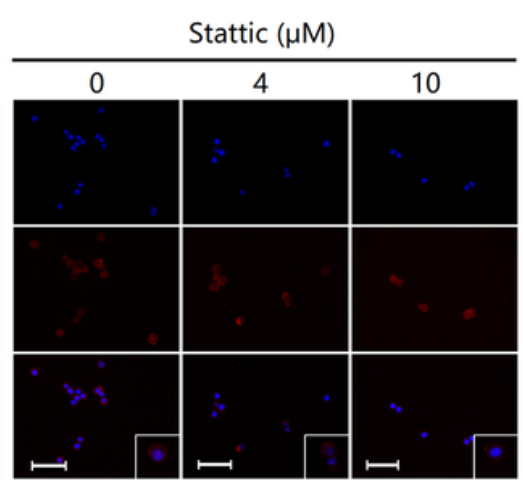

E
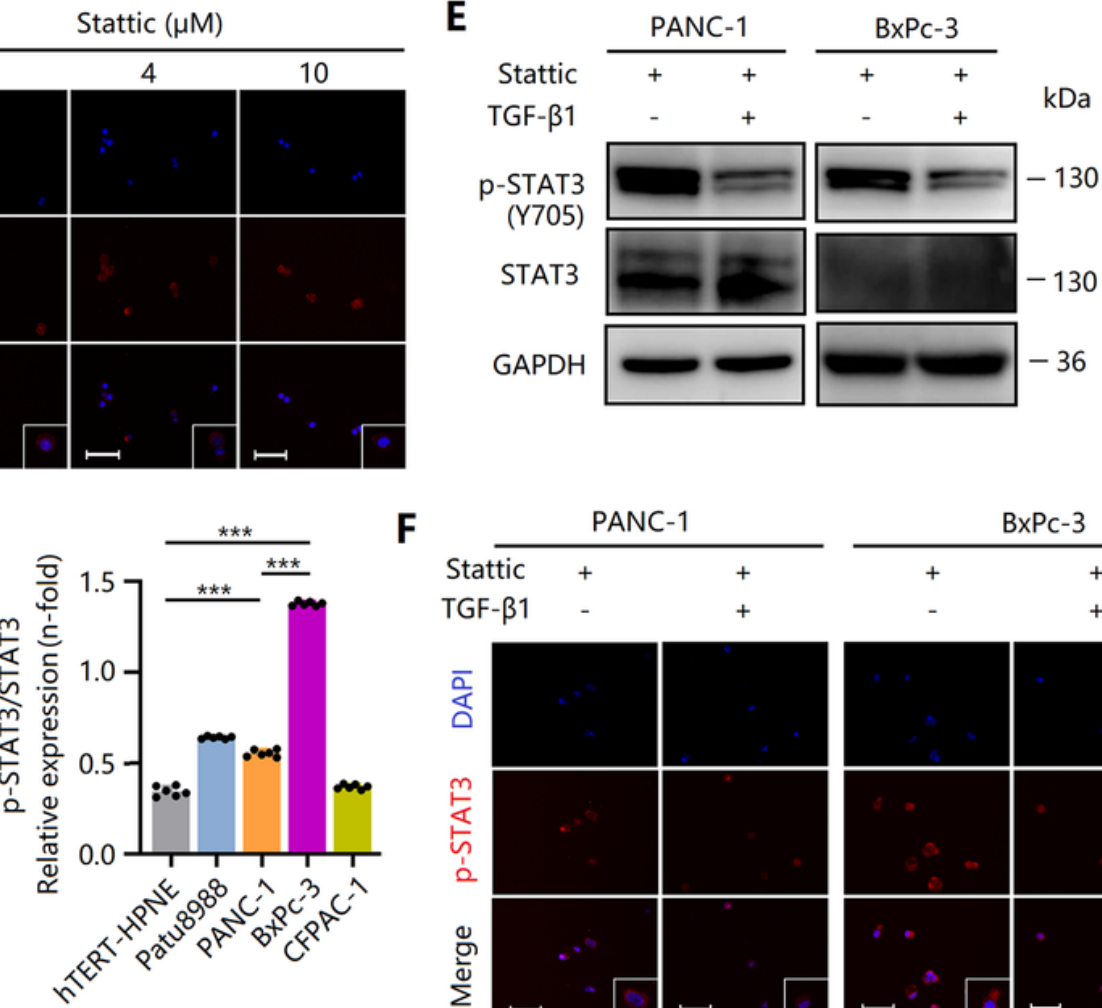

F
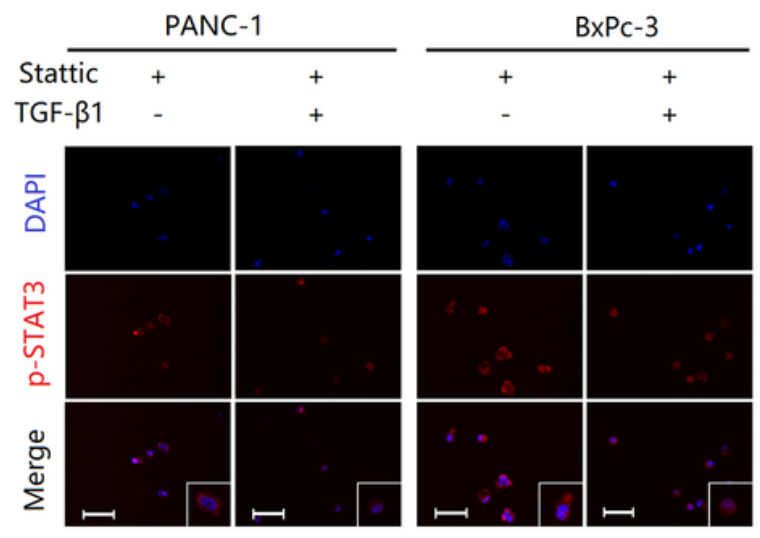

\section{Figure 7}

Stattic inhibits JAK2/STAT3 signaling activation in pancreatic cancer cells. A p-JAK2, JAK2, p-STAT3, and STAT3 western blot results of PANC-1 and BxPc-3 cells after Stattic $(0,4$, and $10 \mu \mathrm{M})$ treatment. B p-JAK2, JAK2, p-STAT3, and STAT3 western blot statistical results of PANC-1 and BxPc-3 cells after Stattic $(0,4$, and $10 \mu \mathrm{M}$ ) treatment. C IF staining of p-STAT3 (red) in PANC-1 and BxPc-3 cells with or without Stattic 
treatment, the nucleus was counterstained with DAPI (blue), bar $=25 \mu \mathrm{m}$. D Western blot results and statistical chart of p-STAT3 and STAT3 protein expression levels in normal human pancreatic ductal epithelial cells and different pancreatic cancer cell lines. E Western blot results of p-STAT3 and STAT3 protein expression in Stattic-treated PANC-1 and BxPc-3 cells with or without TGF- $\beta 1$. F IF staining of $p$ STAT3 (red) in Stattic-treated PANC-1 and BxPc-3 cells with or without TGF- $\beta 1$, the nucleus was counterstained with DAPI (blue), bar $=25 \mu \mathrm{m}$. The experiment was repeated in independent triplicates. Results are expressed as the mean $\pm \mathrm{SD}$. ${ }^{*} * \mathrm{P}<0.001$.
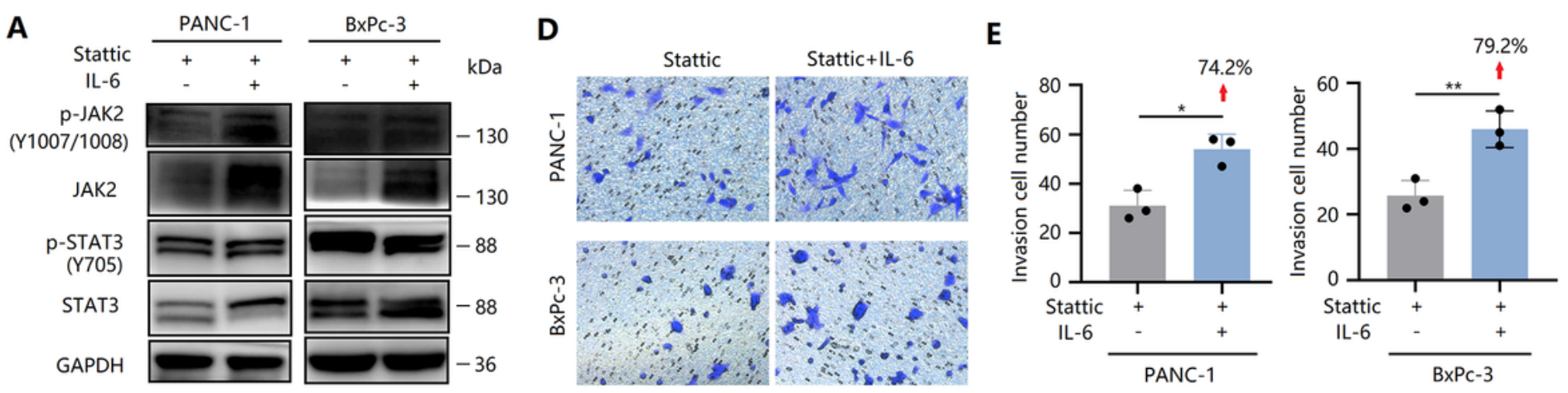

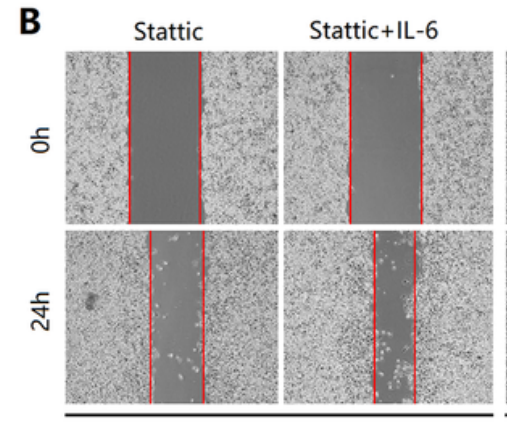

PANC-1
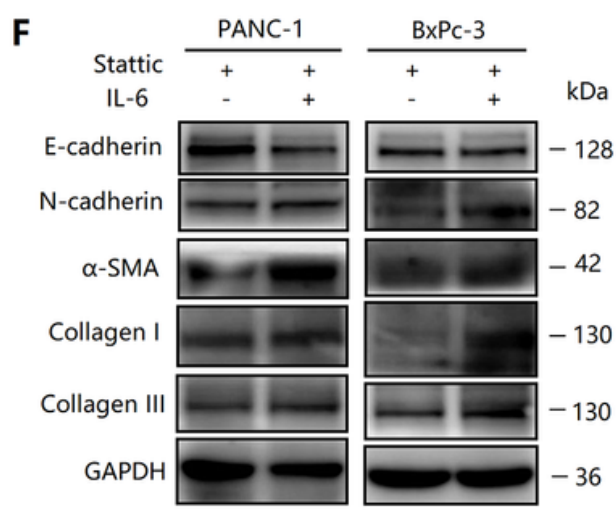

Stattic

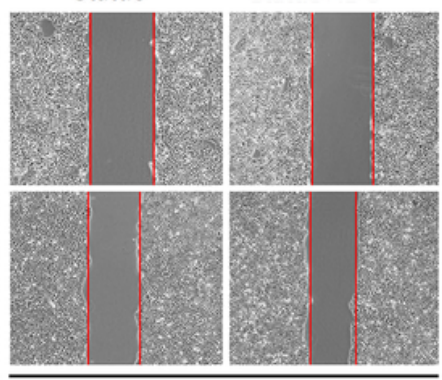

BxPc-3

G

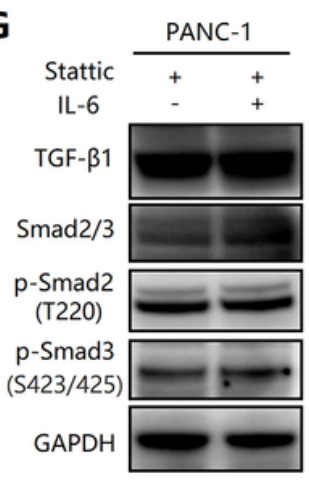

C
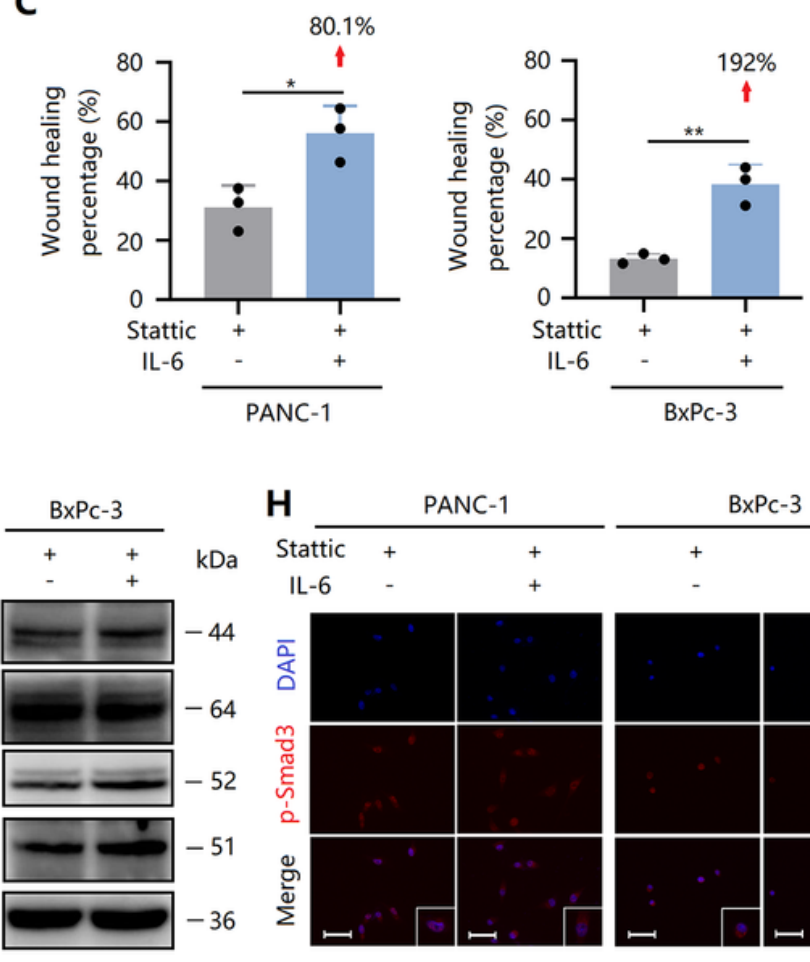
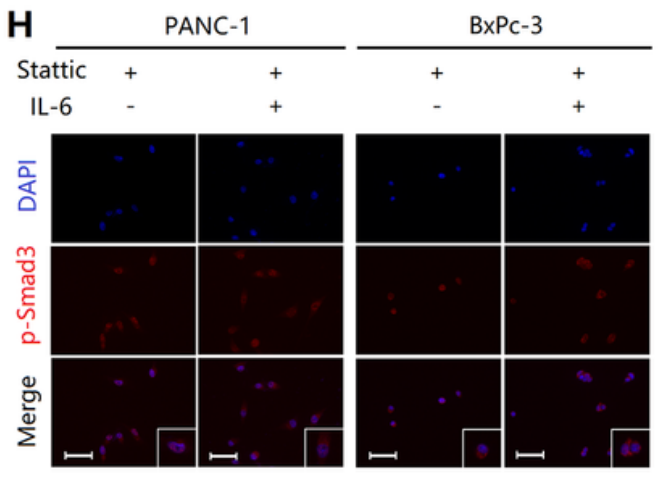

\section{Figure 8}

IL- 6 activates STAT3 to antagonize the inhibitory effects of Stattic on pancreatic cancer cells. A Western blot results of p-JAK2, JAK2, p-STAT3, and STAT3 protein expression in Stattic-treated PANC-1 and BxPc3 cells with or without IL-6. B, C Images and statistical analysis of scratch wound healing assays show that IL- 6 antagonizes the inhibitory effects of Stattic on migration in PANC- 1 and BxPc-3 cells. D, E Images and statistical analysis of Transwell chamber assays show that IL- 6 antagonizes the inhibitory effects of Stattic on invasiveness in PANC-1 and BxPc-3 cells. F Western blot results of EMT-related 
protein expression in Stattic-treated PANC-1 and BxPc-3 cells with or without TGF- $\beta 1$. G Western blot results of TGF- $\beta 1 /$ Smad pathway protein expression in Stattic-treated PANC-1 and BxPc-3 cells with or without TGF- $\beta 1$. H IF staining of p-Smad3 (red) in Stattic-treated PANC-1 and BxPc-3 cell with or without IL-6, the nucleus was counterstained with DAPI (blue), bar $=25 \mu \mathrm{m}$. The experiment was repeated in independent triplicates. Results are expressed as the mean \pm SD. ${ }^{*}<0.05$ and ${ }^{*} \mathrm{P}<0.01$.

A

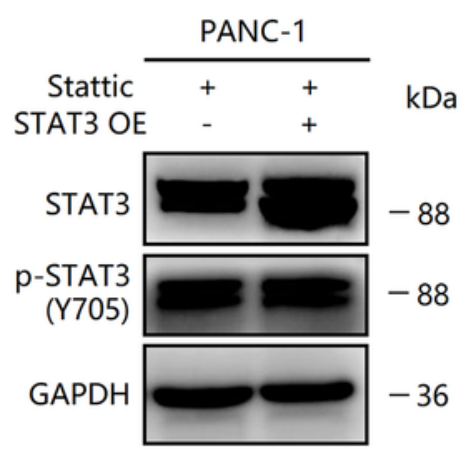

B
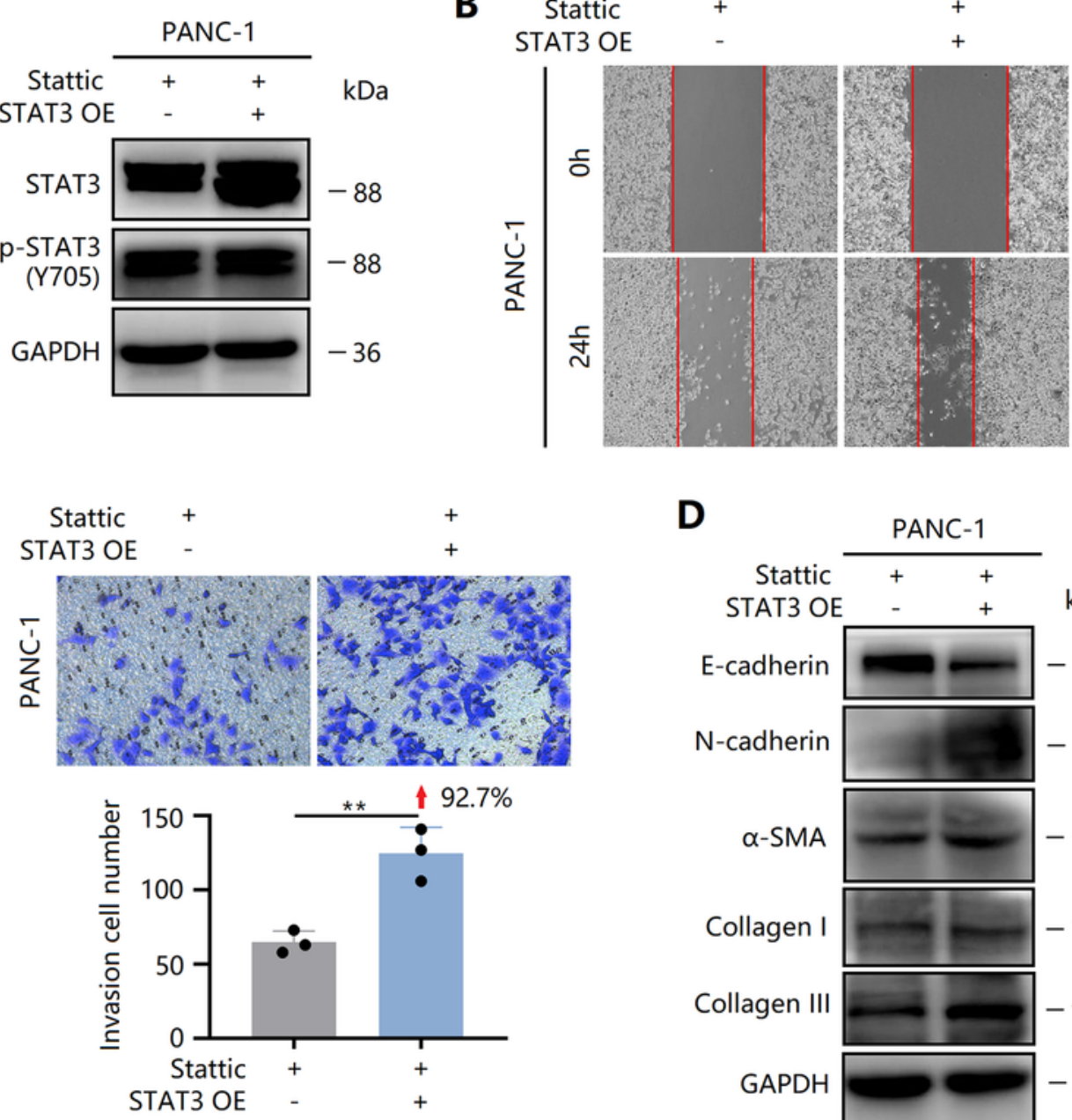

D

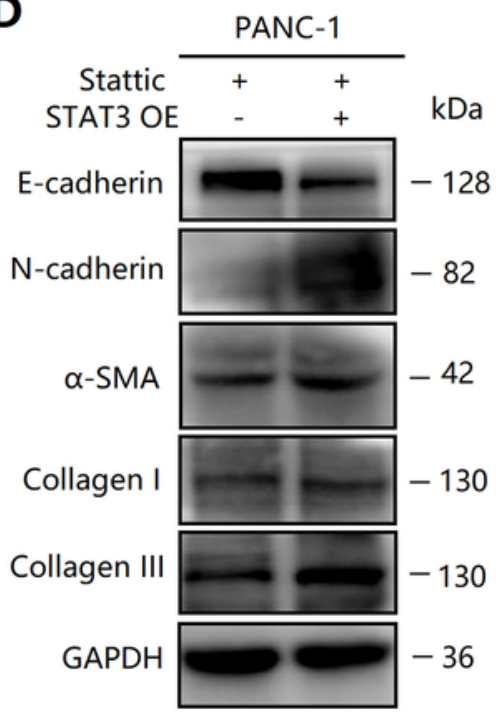

G

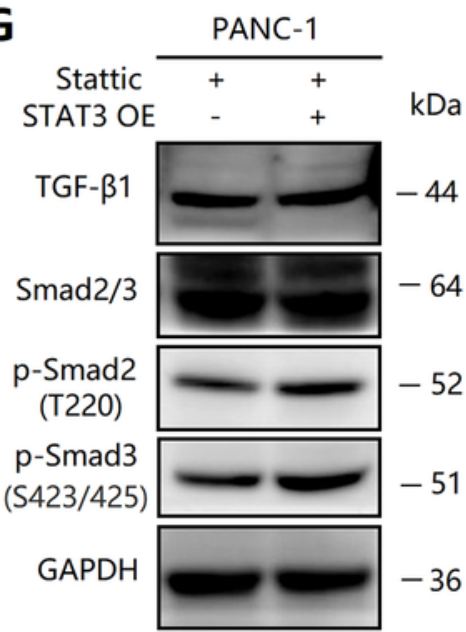

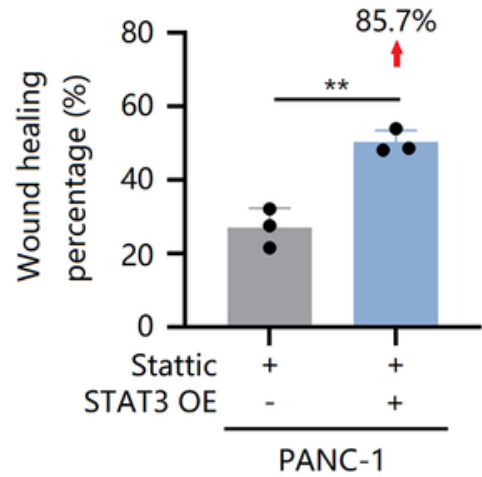

$\mathbf{E}$

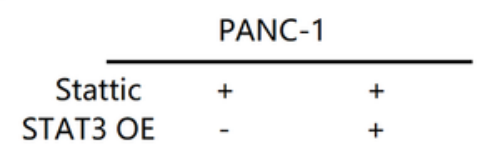

H
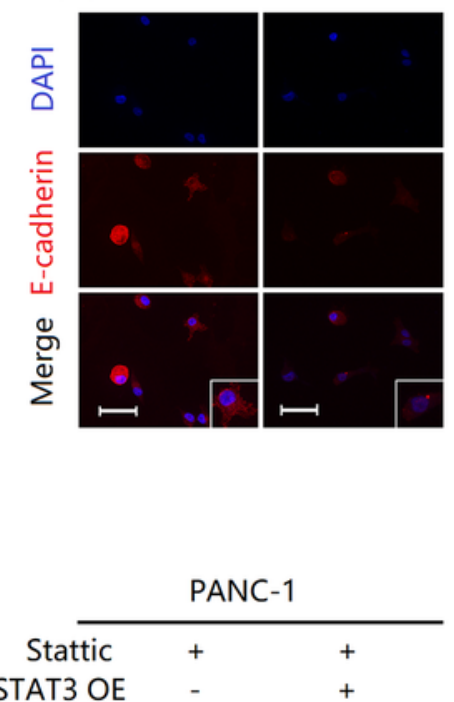
STAT3 OE

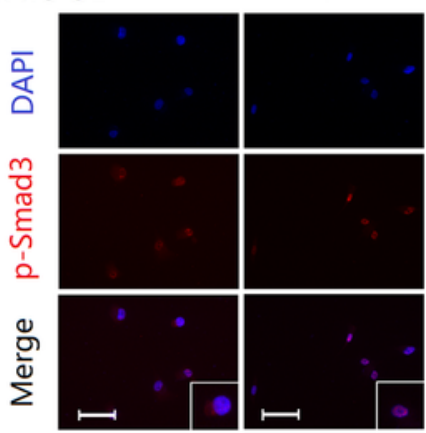

Figure 9 
STAT3 overexpression antagonizes the inhibitory effects of Stattic on pancreatic cancer cells. A Western blot results of p-STAT3 and STAT3 protein expression in Stattic-treated PANC-1 cells with or without STAT3 overexpression. B Images and statistical analysis of the scratch wound healing assays show that overexpression of STAT3 antagonizes the inhibitory effects of Stattic on migration of PANC-1 and BxPc-3 cells. C Images and statistical analysis of Transwell chamber assays show that overexpression of STAT3 antagonizes the inhibitory effects of Stattic on invasiveness in PANC-1 and BxPc-3 cells. D Western blot results of EMT-related protein expression in Stattic-treated PANC-1 cells with or without the STAT3 overexpression. E, F IF staining of E-cadherin (red), a-SMA (red), and collagen III (red) in Stattic-treated PANC-1 cell with or without STAT3 overexpression, the nucleus was counterstained with DAPI (blue), bar = $25 \mu \mathrm{m}$. G Western blot results of TGF- $\beta 1 /$ Smad pathway protein expression in Stattic-treated PANC-1 cells with or without the STAT3 overexpression. H IF staining of p-Smad3 (red) in Stattic-treated PANC-1 cell with or without STAT3 overexpression, the nucleus was counterstained with DAPI (blue), bar $=25 \mu \mathrm{m}$. The experiment was repeated in independent triplicate. Results are expressed as the mean \pm SD. $* \star \star P<0.01$.

\section{Supplementary Files}

This is a list of supplementary files associated with this preprint. Click to download.

- Additionalfile1FigureS1.tif

- Additionalfile2FigureS2.tif

- Additionalfile3FigureS3.tif

- Additionalfile4FigureS4.tif

- Additionalfile5FigureS5.tif

- Additionalfile6FigureS6.tif

- Additionalfile7FigureS7.tif 